\title{
Extraction of Potential Energy from Geostrophic Fronts by Inertial-Symmetric Instabilities $\mathscr{O}$
}

\author{
NICOLAS GRISOUARD \\ Department of Physics, University of Toronto, Toronto, Ontario, Canada
}

(Manuscript received 16 August 2017, in final form 8 February 2018)

\begin{abstract}
Submesoscale oceanic density fronts are structures in geostrophic and hydrostatic balance, which are prone to inertial and/or symmetric instabilities. We argue in this article that drainage of potential energy from the geostrophic flow is a significant source of their growth. We illustrate our point with two-dimensional Boussinesq numerical simulations of oceanic density fronts on the $f$ plane. A set of two-dimensional initial conditions covers the submesoscale portion of a three-dimensional parameter space consisting of the Richardson and Rossby numbers and a measure of stratification or latitude. Because we let the lateral density gradient decay with depth, the parameter space map is nontrivial, excluding low-Rossby, low-Richardson combinations. Dissipation and the presence of boundaries select a growing mode of inertial-symmetric instability consisting of flow cells that disturb isopycnal contours. Systematically, these isopycnal displacements correspond to a drainage of potential energy from the geostrophic fronts to the ageostrophic perturbations. In the majority of our experiments, this energy drainage is at least as important as the drainage of kinetic energy from the front. Various constraints, some physical, some numerical, make the energetics in our experiments more related to inertial rather than symmetric instabilities. Our results depend very weakly on the Richardson number and more on the Rossby number and relative stratification.
\end{abstract}

\section{Introduction}

Oceanic density fronts are defined by horizontal density gradients that are relatively strong over a relatively narrow region of the ocean surface. Here, we focus on fronts that are in geostrophic balance and that decay with depth from the ocean surface. Thermal wind balance implies that a vertical gradient in geostrophic velocity exists within the front. Integrating this shear from depth where currents are weak results in a surfaceintensified jet along the front (Hoskins 1975).

A front is said to be submesoscale if its Rossby and Richardson numbers are both of order one; that is, respectively,

$$
\overline{\mathrm{Ro}}=\frac{\partial_{x} \bar{v}}{f}=O(1) \quad \text { and } \quad \overline{\mathrm{Ri}}=\frac{N^{2}}{\left|\partial_{z} \bar{v}\right|^{2}}=O(1),
$$

Supplemental information related to this paper is available at the Journals Online website: https://doi.org/10.1175/JPO-D-170160.s1.

Corresponding author: Nicolas Grisouard, nicolas.grisouard@ physics.utoronto.ca where $\bar{v}$ is the geostrophic jet velocity, assumed to flow along the $y$ direction, $x$ is the across-front direction, and $z$ is the vertical direction, oriented positively upward. The Coriolis frequency is $f$, equal to twice Earth's angular velocity multiplied by the sine of the local latitude (assumed constant in this study). The buoyancy frequency is $N=\sqrt{-\left(g / \rho_{0}\right) \partial_{z} \bar{\rho}}, g$ is the gravitational acceleration, and $\rho_{0}$ is a reference density, equal to $1025 \mathrm{~kg} \mathrm{~m}^{-3}$ in the rest of this article. Finally, $\bar{\rho}$ is the unperturbed density field in thermal wind balance with $\bar{v}$. Note that the definition of $N$ implies that we use the Boussinesq approximation, which holds well for such flows, and that the Rossby number is allowed to take negative values. At midlatitudes, $\overline{\mathrm{Ro}}$ and $\overline{\mathrm{Ri}}$ being of order one translates into submesoscale fronts that are on the order of 1 to $10 \mathrm{~km}$ wide and a few meters to tens of meters deep.

Submesoscale fronts host processes that intensely dissipate kinetic energy (KE) (Capet et al. 2008; Molemaker et al. 2010; D'Asaro et al. 2011; Whitt and Thomas 2015; Grisouard and Thomas 2016), which may be significant in closing of the KE budget of the oceans (e.g., Ferrari and Wunsch 2009; McWilliams 2016). This is partially due to their susceptibility to instabilities (Boccaletti et al. 2007; 
Ménesguen et al. 2012; Wang et al. 2014; Arobone and Sarkar 2015) and, in particular, inertial and symmetric instabilities (InI and SI, respectively) (Stone 1966; Cushman-Roisin and Beckers 2011). InI and SI occur in the oceanic mixed layer (Haine and Marshall 1998; Taylor and Ferrari 2009; Thomas et al. 2013) and at low latitudes (e.g., Dunkerton 1981; Griffiths 2003a,b; D’Orgeville and Hua 2005; Colin de Verdière 2012; Natarov and Richards 2015), where the Ertel potential vorticity (EPV) is of small magnitude owing to the weak stratification or small $|f|$. There, it can relatively easily change sign as a result of the addition or removal of EPV by wind stress and/or buoyancy fluxes. When the sign of the EPV becomes opposite to that of $f$, the front may become unstable with respect to InI and/or SI. Note that the precise definitions of InI and SI, and how to distinguish them, vary across different groups of authors. The ones we are about to use follow that of, for example, Haine and Marshall (1998) or Taylor and Ferrari (2009). In particular, Thomas et al. (2013) propose a systematic way to define them, based on their energetic signatures.

The first limit case, the "pure InI" case, is that of a barotropic geostrophic flow with uniform anticyclonic shear characterized by $\overline{\mathrm{Ro}}<-1$. In this case, the flow is susceptible to InI, which feeds off of the KE of the laterally sheared flow. The KE of the perturbations increases as a result of lateral shear production:

$$
\mathrm{LSP}=f \overline{\operatorname{Ro}} u v,
$$

where $u$ is the across-front, horizontal velocity, and $v$ are the fluctuations in along-jet velocity (with $\bar{v}+v$ the total along-jet velocity).

The second extreme, the "pure SI" case, is that of a baroclinic, geostrophic flow with uniform lateral density gradient (an infinitely wide density front) and therefore uniform thermal wind shear. The necessary condition for pure SI is that $\overline{\mathrm{Ri}}<1$. More generally, SI occurs when $\overline{\mathrm{Ro}}>-1$, in which case the necessary condition for instability is $\overline{\mathrm{Ri}}<1 /(1+\overline{\mathrm{Ro}}$ ) (Haine and Marshall 1998; Thomas et al. 2013). SI feeds off of the KE of the vertically sheared geostrophic flow. The KE of the perturbations increases as a result of geostrophic shear production:

$$
\mathrm{GSP}=\frac{M^{2}}{f} v w
$$

where $w$ is the vertical velocity and $M^{2}=-\left(g / \rho_{0}\right) \partial_{x} \bar{\rho}$ is the lateral buoyancy gradient.

When shifting our focus away from these energetic considerations, however, the two instabilities are strikingly similar. For the purpose of the present article, we do not always need to emphasize the difference between the two instabilities, and therefore, we often refer to one "inertial-symmetric instability" (ISI), which encompasses the spectrum of instabilities, from one "pure" form to the other.

When considered within the most idealized linear stability analysis (i.e., in an infinite domain with constant coefficients), these instabilities only affect the KE budget of the geostrophic flow. Indeed, InI is essentially barotropic in nature, while SI's and ISI's fastest-growing modes flow along isopycnals. It is therefore customary to think of these instabilities as only marginally affecting the potential energy (PE) of geostrophic fronts.

While not the main topic of the current article, a few words should be said about scale selection of the dominant growing mode. This topic is still actively researched, but results from linear stability analysis predict that the scale of the fastest-growing mode is the result of a competition between two processes. On one hand, in any realistic setting, ISI develops in a finite region of space, bounded by the zero EPV contour, and sometimes by a rigid boundary such as the ocean surface in our case. The imposition of such boundaries in an inviscid fluid is thought to be associated with an "ultraviolet catastrophe"; that is, the growth rate increases monotonously with the wavenumber. It does so asymptotically, toward a finite value, which is that of the along-isopycnal perturbation in an unbounded domain mentioned previously. On the other hand, diffusion of momentum and tracers stabilizes the shortest scales. This competition has been studied extensively for pure InI (e.g., Dunkerton 1981; Kloosterziel and Carnevale 2008; Griffiths 2008) but applies also to pure SI (Stone 1966; Griffiths 2017).

One point worth noting is that the stabilization of the shorter scales by diffusive processes does not necessarily imply that the fastest-growing mode is "viscous"; that is, its growth rate does not have to be significantly impacted by the diffusive time scale. A manifestation of this is that the peak of the growth rate as a function of wavenumber does not have to be sharp and that for small enough diffusivities, a relatively large number of modes can grow at about the same speed (Dunkerton 1981; Griffiths 2017). Another point worth noting is that in the case of InI in the atmosphere, linear stability analysis significantly underestimates the scales observed (Griffiths 2003b). Finally, numerical simulations of pure SI occurring in oceanic submesoscale fronts systematically observe that the fastest-growing scale is the largest one that the size of the unstable region can accommodate (e.g., Thomas 2005; Bachman and Taylor 2014), even when viscosity is low enough to resolve secondary instabilities (Taylor and Ferrari 2009).

To study the processes that set the scale of the ISI flow is outside the scope of the current article, and whatever they 
are, we hereinafter refer to their outcome as "diffusive scale selection" (DSS). To our knowledge, no stability analysis, linear or not, has been done for ISI at the level of detail that is available for pure InI or even pure SI. Nonetheless, we will keep discussing the potential influence of diffusive processes on our results when appropriate.

In this article, we instead focus on the consequences of ISI. In particular, we observe that contrary to the usual argument mentioned earlier, its outcome is a flow that creates significant PE-extracting diapycnal velocities. In fact, the main result of this article is that in such a configuration, the instabilities feed off of the PE of the front in a significant way and turn it into PE fluctuations. Furthermore, the term that executes the PE transfers cannot be attributed to either pure InI or pure SI, which supports our choice of often referring to one ISI.

In the present study, we retain a large degree of idealization, in the sense that we will only consider twodimensional fronts $\left(\partial_{y} \equiv 0\right)$ and that we will consider initial value problems, rather that generating the fronts with realistic forcing. We adopt a heuristic approach based on numerical experiments, with the intention to motivate future analytical analyses such as that of Ménesguen et al. (2012).

An important intermediate concept we introduce in this article is that of a "geometric exclusion regime" (GXR). Its existence derives from $N^{2}$ having to be initially positive everywhere and is explained in the appendix. The extent of the GXR will vary for different frontal shapes, but its consequence is relatively simple: low Richardson, low Rossby numbers (in terms of magnitude) are difficult to find in tandem.

This article is organized as follows. In section 2, we present some basic theoretical facts about ISI. In section 3, we introduce the setup of our experiments, as well as the GXR, and present our road map to the parameter space. In section 4, we present a numerical illustration of the process at stake and lay out our methodology to interpret the energy budgets of the experiments. To ease us into the analysis of the full experimental set, section 5 presents two additional case studies, illustrating cases that resemble either pure InI or pure SI. Building upon all previous sections, in section 6, we propose a systematic analysis of all our numerical experiments, discuss the importance of a three-parameter description of ISI, and notice the relative lack of relevance of $\overline{\mathrm{Ri}}$ and GSP to understand the energetics of ISI. We present our conclusions are some perspectives in section 7 .

\section{Review of potential vorticity and linear stability}

ISI happens when the EPV and $f$ are of opposite signs, namely, when $f \bar{Q}=f \nabla \bar{b} \cdot(f \hat{\mathbf{z}}+\nabla \times \bar{v} \hat{\mathbf{y}})=f^{2} N^{2}(1+\overline{\mathrm{Ro}}-1 / \overline{\mathrm{Ri}})<0$.

In the equation above, $\bar{b}=-g\left(\bar{\rho}-\rho_{0}\right) / \rho_{0}$ is the unperturbed buoyancy field and $\hat{\mathbf{x}}, \hat{\mathbf{y}}$, and $\hat{\mathbf{z}}$ are the acrossflow, along-flow, and vertical unit vectors of a positive Cartesian system of coordinates. For future reference, $\hat{\mathbf{z}}$ points upward, with the ocean surface located at $z=0$. The occurrence of $1 / \overline{\mathrm{Ri}}$ in Eq. (4) derives from inserting the thermal wind balance relation

$$
\frac{\partial \bar{v}}{\partial z}=\frac{1}{f} \frac{\partial \bar{b}}{\partial x}
$$

into Eq. (1).

For a confined front, $\bar{Q}$ varies in space (e.g., Fig. 1c). For now, however, we briefly ignore the spatial variations of $N^{2}, \overline{\mathrm{Ri}}$, and $\overline{\mathrm{Ro}}$, and the existence of boundaries. All coefficients of the equations of motion are then constant and a simple linear stability analysis provides a useful qualitative description of the growing perturbations. We consider a two-dimensional framework where no along-flow perturbations are allowed $\left(\partial_{y} \equiv 0\right)$. The linear, hydrostatic, inviscid Boussinesq equations, with the momentum equation expanded around $\bar{v}$ and the thermodynamic equation expanded around $\bar{b}$, are as follows (e.g., Whitt and Thomas 2013):

$$
\begin{aligned}
\partial_{t} u-f v+\partial_{x} p & =0, \\
\partial_{t} v+f(1+\overline{\mathrm{Ro}}) u+M^{2} w / f & =0, \\
-b+\partial_{z} p & =0, \\
\partial_{t} b+M^{2} u+N^{2} w & =0, \quad \text { and } \\
\partial_{x} u+\partial_{z} w & =0 .
\end{aligned}
$$

Recall that $1 / \overline{\mathrm{Ri}} \neq 0$ whenever $M^{2} \neq 0$. The velocity vector $\mathbf{v} \equiv u \hat{\mathbf{x}}+v \hat{\mathbf{y}}+w \hat{\mathbf{z}}$ represents the deviations about $\bar{v} \hat{\mathbf{y}}, b$ is the buoyancy fluctuation field about $\bar{b}$, and $p$ is the scaled pressure. Temporarily ignoring the presence of boundaries allows us to consider plane waves; namely, $\{\mathbf{v}, b\}=\left\{\mathbf{v}_{0}, b_{0}\right\}$ expi $[k x+m z-(\omega+i \sigma) t]$, where $k, m$, $\omega$, and $\sigma$ are all real numbers. Canceling the determinant of the resulting linear system of equations yields:

$$
\omega+i \sigma= \pm \sqrt{f \bar{Q} / N^{2}+N^{2}(\mu+\beta)^{2}},
$$

where $\beta=M^{2} / N^{2}$ and $\mu=-k / m$. When the right-hand side of Eq. (7) is purely real, we recover the dispersion relation for internal waves in a baroclinic geostrophic flow (Whitt and Thomas 2013). When $f \bar{Q}<0$ on the other hand, one recovers the growth rate of ISI (e.g., Taylor and Ferrari 2009). In this case, indeed, at least 

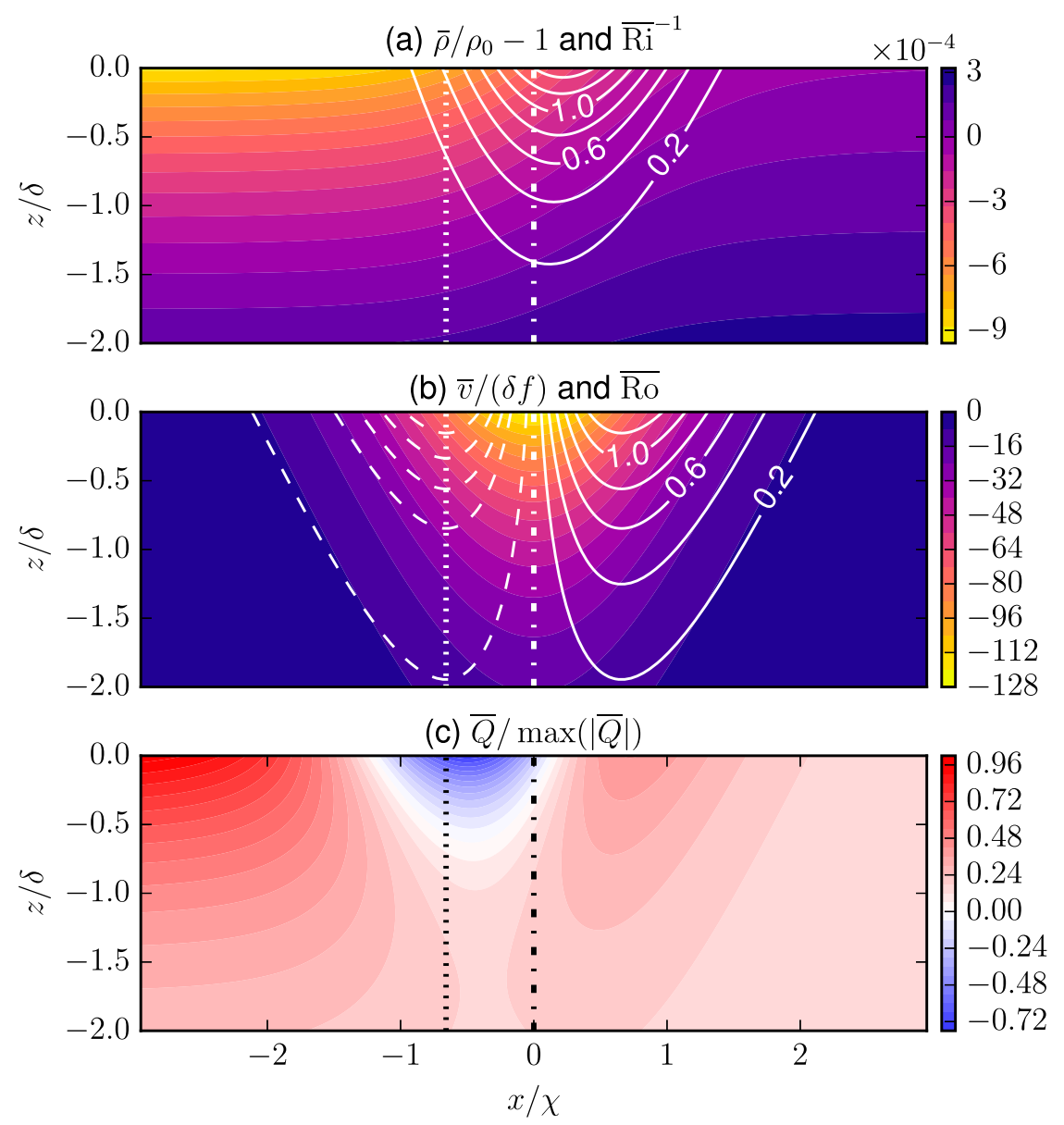

FIG. 1. Sketch of an unstable front, with $\lambda=(1-\tanh ) / 2, \Phi_{0}=50$, and $-\operatorname{Ro}_{0}=1 / \operatorname{Ri}_{0}=1.4$. In all figures, the dashed-dotted line marks the location $x_{i}$ where $M^{2}$ is maximum and the dotted line marks the location $x_{o}$ where $\overline{\mathrm{Ro}}$ is minimum. (a) Density $\bar{\rho} / \rho_{0}-1=-\bar{b} / g$ (shading) and $1 / \overline{\mathrm{Ri}}$ (one white contour every 0.2 ). (b) Geostrophic flow $\bar{v}$ (shading) and $\overline{\mathrm{Ro}}$ (one white contour every 0.2 ; dashes indicate negative values, $\overline{\text { Ro }}$ being axially symmetric with respect to $x=0$ ). (c) EPV $\bar{Q}$, exhibiting a negative region where ISI can grow.

one perturbation mode is unstable, namely, the one for which $\mu=-\beta$, in which case $\omega=0$ and

$$
\sigma_{0}=|f| \sqrt{\overline{\mathrm{Ri}}^{-1}-\overline{\mathrm{Ro}}-1} .
$$

This particular mode, whose motions are aligned with isopycnals, is in fact the fastest-growing mode in the simple case we are describing. Because it does not disturb isopycnals, we only expect this instability to manifest itself in terms of kinetic energy. Colin de Verdière (2012) and Bachman and Taylor (2014) notice that if the focus shifts away from this along-isopycnal, fastest-growing mode, ISI can in principle extract PE from fronts. However, they do not provide scenarios as to why the fastestgrowing mode would not manifest itself primarily.

Indeed, according to Eq. (7), for a given geostrophic flow, only the orientation $\mu$ of the flow perturbations determines the growth rate, not its scale (Taylor and Ferrari 2009; Colin de Verdière 2012). However, recall from section 1 that if we include diffusion of momentum and/or buoyancy in the equations, and if we take into account the boundaries of the unstable region, ISI selects one mode of motion under what we call DSS. We can reasonably expect that the results from linear stability analysis of pure InI and pure SI, as well as the caveats associated with them (see section 1), can be extended to ISI. If so, the choice of diffusive operators may have consequences for the scale selection and, therefore, quantitative consequences for our results. It is hard to predict what this influence will be, and we prefer to adopt a more pragmatic approach, which is to identify and keep track of the potential consequences of the diffusion operators, in a diagnostic manner.

We use frictional operators of the form 


$$
\mathcal{D}=\nu_{h} \partial_{x}^{2}+\nu_{z} \partial_{z}^{2}+\nu_{3 h} \partial_{x}^{6},
$$

which will apply to both the momentum and thermodynamic equations at the same time. In Eq. (9), $\nu_{h}, \nu_{z}$ and $\nu_{3 h}$ are the horizontal harmonic, vertical harmonic, and horizontal tri-harmonic diffusion coefficients, respectively. The inverse time scale for the dissipation of a given plane wave mode $(k, m)$ is $\tau_{\mathcal{D}}=\nu_{h} k^{2}+\nu_{z} m^{2}+\nu_{3 h} k^{6}$. When simulating ISI with a given number of grid points, we employ diffusion coefficients that are as small as possible for the initial motion, and verify that

$$
\alpha^{2}=\tau_{\mathcal{D}} / \sigma_{0} \ll 1
$$

Even with very low values of $\alpha^{2}$, in all of our simulations, the fastest-growing mode that emerges is typically one with a scale, comparable with that of the entire unstable region, consistent with the results of other authors (Thomas 2005; Taylor and Ferrari 2009). We further discuss reasons and consequences of choosing this particular operator in section $3 \mathrm{~b}$, and in section $6 \mathrm{~d}$, we investigate this point further and see that diffusive processes, as we were able to quantify them, do not seem to noticeably impact our results.

\section{Setup}

\section{a. Fundamental parameters}

A computation of the unstable eigenmodes in a domain with boundaries, such as that of Ménesguen et al. (2012), might yield the exact growth rate of the instability, but would require a separate study. We prefer to use the simple results from the previous section to guide the design of our numerical experiments. We first define an initial buoyancy field:

$$
\bar{b}=N_{0}^{2} z+B \lambda(x / \chi) \exp (z / \delta),
$$

where $N_{0}$ is the background Brunt-Väisälä frequency, $B$ is the buoyancy difference across the front, $\lambda$ is the horizontal structure of the buoyancy variations, $\chi$ is the characteristic width of the front, and $\delta$ is the $e$-folding depth scale over which the front penetrates (see Fig. 1a).

Integrating Eq. (5) from an infinite depth yields the geostrophic velocity:

$$
\bar{v}=\frac{B \delta}{f \chi} \lambda^{\prime}\left(\frac{x}{\chi}\right) \exp \left(\frac{z}{\delta}\right),
$$

where the prime denotes the exact derivative (see Fig. 1b).

Except for nondimensional measures of viscous effects, which are usually kept as small as possible, several idealized studies of submesoscale dynamics only explore one nondimensional parameter, usually the Richardson or Rossby number. To achieve such a simplification, assumptions have to be made, for example by ignoring nontrivial spatial structures of the geostrophic flow (e.g., Stone 1966; Grisouard and Thomas 2016) or baroclinic effects (e.g., Plougonven and Zeitlin 2009). We make no such simplifications. Our initial density and flow fields require the introduction of five independent dimensional quantities, $N_{0}, B, \chi, \delta$, and $f$, measured in combinations of distance and duration. Therefore, we need three independent nondimensional parameters to characterize these initial conditions.

First, we choose representative values of the minima of $\overline{\mathrm{Ri}}$ and $\overline{\mathrm{Ro}}$, so that they indicate if the initial condition is unstable. For the Rossby number, we define (see the appendix)

$$
\operatorname{Ro}_{0}=\overline{\operatorname{Ro}}\left(x=x_{o}, z=0\right),
$$

where $x_{o}$ is defined such that $\lambda^{\prime \prime \prime}\left(x_{o} / \chi\right)=0$ and $\lambda^{\prime \prime}\left(x_{o} / \chi\right)<0$. That is, $x_{o}$ is at the location of maximum anticyclonicity. Similarly, we define

$$
\mathrm{Ri}_{0}=\overline{\operatorname{Ri}}\left(x=x_{i}, z=0\right),
$$

where $x_{i}$ is defined such that $\lambda^{\prime \prime}\left(x_{i} / \chi\right)=0$, that is, at the center of the jet. Contrary to what $\mathrm{Ro}_{0}$ is to $\overline{\mathrm{Ro}}$, $\mathrm{Ri}_{0}>\min (\overline{\mathrm{Ri}})$ because the location of $\min (\overline{\mathrm{Ri}})$ is always slightly shifted toward the cyclonic flank of the jet (see the white contours in Fig. 1a). Nonetheless, it is close enough for our purpose.

Perhaps the most surprising outcome of this procedure is the existence of a GXR, which originates from the constraint that $N^{2}>0$ everywhere in the domain; that is, initial isopycnal contours never cross (see the appendix). ${ }^{1}$ The primary culprit is the introduction of either a vertical decay scale $\delta$ or horizontal width $\chi$, this effect completely disappearing when either $\delta \rightarrow \infty$ or $\chi \rightarrow \infty$. However, oceanic fronts do have a finite size, which is likely to play a role because of diffusive scale selection. The main consequence is that $\mathrm{Ri}_{0}$ and $\mathrm{Ro}_{0}$ have to satisfy the following [see Eq. (A7)]:

$$
\operatorname{Ri}_{0} \operatorname{Ro}_{0}<\hat{a}=\frac{\lambda\left(x_{i} / \chi\right) \lambda^{\prime \prime}\left(x_{o} / \chi\right)}{\left[\lambda^{\prime}\left(x_{i} / \chi\right)\right]^{2}}
$$

The inequality above reflects a geometric constraint of our setup, with $\chi^{\prime} \delta \rightarrow \infty$ as $\mathrm{Ri}_{0} \mathrm{Ro}_{0} \rightarrow \hat{a}$. Combinations

\footnotetext{
${ }^{1}$ To illustrate this nontrivial constraint, we provide a Python script in the supplemental material, which plots the initial conditions for a given set of $\mathrm{Ri}_{0}, \mathrm{Ro}_{0}$, and $\Phi_{0}$.
} 


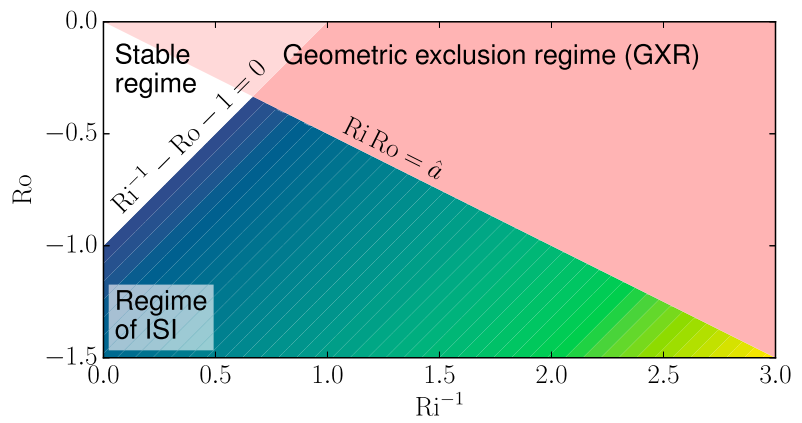

FIG. 2. Sketch of the (Ri, Ro) parameter space. For illustration purposes, $\hat{a}=-1 / 2$. In white is the stable regime for which the EPV and $f$ are of opposite sign. In red is the GXR, that is, the couples ( $\mathrm{Ri}, \mathrm{Ro}$ ) that violate Eq. (15). Shaded are the couples (Ri, Ro), which correspond to a possible unstable front, color coded by $\mathrm{Ri}^{-1}-\mathrm{Ro}-1$. Note that this figure is merely a sketch: $\mathrm{Ri}$ and $\mathrm{Ro}$ do not correspond to $\mathrm{Ri}_{0}$ and $\mathrm{Ro}_{0}$, and $\hat{a} \neq-1 / 2$ in the text.

for which $\mathrm{Ri}_{0} \mathrm{Ro}_{0}>\hat{a}$ are in the GXR, as illustrated in Fig. 2. The right-hand side of Eq. (15), which happens to be always negative here, only depends on the function used to define the lateral structure of the front, with $x_{i} / \chi$ and $x_{o} / \chi$ being mere consequences of this choice. However, a few tries with the usual smooth jump functions (tanh, erf, $1-\mathrm{e}^{-x^{2}}$, etc.) all yield results in the range -0.6 to -0.8 .

One of the consequences of the GXR is that a pure SI regime, as defined in the introduction, is hard to reach. Indeed, to tip the scale from InI to SI, one needs both $\mathrm{Ri}_{0}$ and $\left|\mathrm{Ro}_{0}\right|$ to be small, which is less likely to satisfy Eq. (15) (recall that both sides of the inequality are negative, since $\mathrm{Ro}_{0}<0$ by definition). How much the GXR is likely to apply in nature, versus a pure artifact of our setup, will not be investigated further.

Finally, for the third nondimensional parameter, we choose an inverse Prandtl ratio $^{2}$ (Vallis 2006, p. xiv); namely,

$$
\Phi_{0}=N_{0} / f
$$

Equivalently, one could choose the aspect ratio $\chi / \delta$ [see Eq. (A5)]. The $\Phi_{0}$ can be interpreted in several ways, with a high value translating into, for example, a lowlatitude, high-stratification, and/or shallow aspect ratio. It adds a third dimension to the parameter space. Multiple authors consider a low stratification $\left(\right.$ low $\left.\Phi_{0}\right)$ to be a prerequisite for enhanced submesoscale instability activity (Thomas et al. 2013; Callies et al. 2015; McWilliams 2016), while others treat the low latitudes

\footnotetext{
${ }^{2}$ This is not to be confused with the Prandtl number, the ratio of momentum versus buoyancy diffusivity.
}

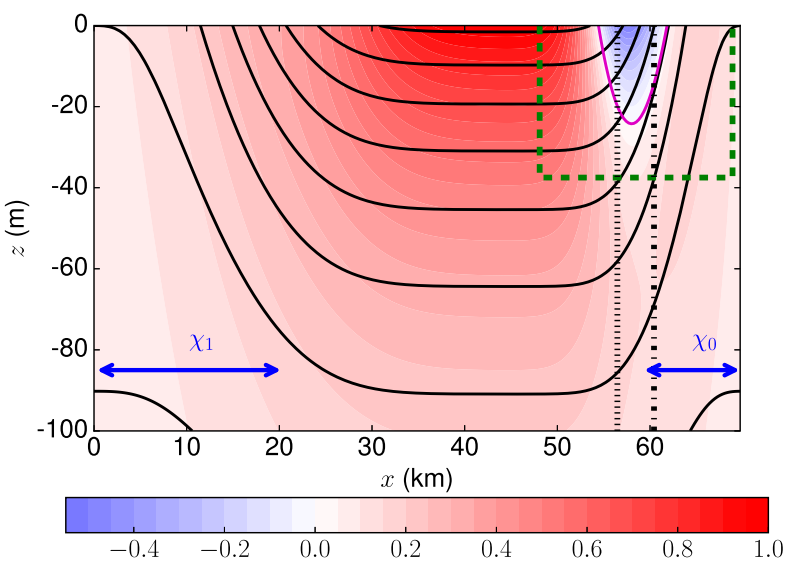

FIG. 3. Initial condition and physical domain for our numerical simulations, for $\mathrm{Ro}_{0}=-1.2, \mathrm{Ri}_{0}^{-1}=1.4$ and $\Phi_{0}=50$, in which case the domain is a size M. Shading represents $\bar{Q} / \max (\bar{Q})$, the solid magenta line highlights the $\bar{Q}=0$ contour, and black solid lines are density contours. As in Fig. 1, the dashed-dotted and dotted lines mark the locations $x_{o}$ and $x_{i}$, respectively. The green dashed rectangle shows the boundaries of the control volume used in the energy budget of section 4 , and the blue double arrows show the widths $\chi_{0}$ and $\chi_{1}$.

(high $\Phi_{0}$ ) as fertile ground for ageostrophic instabilities (Dunkerton 1981; D'Orgeville and Hua 2005; Holmes et al. 2014; Natarov and Richards 2015). However, to our knowledge, this parameter has not been treated as systematically as Ro or $\mathrm{Ri}$ to characterize the submesoscale, other than this role as a prerequisite. We will explore the parameter space along these three axes.

For a given choice of $\lambda$, each numerical experiment is identified by $\mathrm{Ro}_{0}, \mathrm{Ri}_{0}$, and $\Phi_{0}$. One last set of nondimensional numbers should complete the description of our problem, namely, those related to frictional effects, which keep the numerical simulations stable. However, we will keep these effects as small as possible in the initial stages of the simulations. Because our focus is on how ISI extracts energy from geostrophic flows, and because dissipation of the perturbations appears to be only a consequence of this extraction, we defer a discussion on frictional effects to section $6 \mathrm{~d}$.

\section{b. Physical and numerical configurations}

The physical setup is sketched in Fig. 3. Our domains come in four horizontal sizes: XS, S, M, and L, of horizontal lengths $L_{x}=17.5,35,70$, and $140 \mathrm{~km}$, respectively. Indeed, as it turns out, $\chi$ tends to infinity as $\mathrm{Ri}_{0} \mathrm{Ro}_{0}$ approaches $\hat{a}$ [cf. Eqs. (15) and (A5)]. Thus, a numerical experiment that approaches this limit requires a larger domain than an experiment that does not. In all numerical experiments, our domain is $L_{z}=100 \mathrm{~m}$ deep, $\delta=50 \mathrm{~m}$, and $f=1.03 \times 10^{-4} \mathrm{~s}^{-1}$, with $T_{f}=2 \pi / f=61000 \mathrm{~s}$. 
The code we use, a modification of that of Winters et al. (2004), is horizontally periodic, which necessitates setting up initial conditions which are also periodic. We choose

$\lambda=\lambda_{i}\left(\frac{L_{x}-x}{\chi_{0}}\right) \lambda_{i}\left(\frac{x}{\chi_{1}}\right), \quad$ with $\quad \lambda_{i}(\xi)=1-\mathrm{e}^{-|\xi|^{3}}$.

The function $\lambda$ above consists of two fronts of opposite signs and of widths $0<\chi_{0}<\chi_{1}$, such that $\exp \left[-\left(L_{x} / c_{0}\right)^{3}\right]<\exp \left[-\left(L_{x} / c_{1}\right)^{3}\right]=1$. This ensures horizontal periodicity of $\bar{b}$ and $N^{2}$, with $\left.\lambda\right|_{x=0}=0=\left.\lambda\right|_{x=L_{x}}$. Separating the two fronts also ensures that they do not affect each other's shape. All results that follow are derived in the appendix. The $M^{2}, \overline{\mathrm{Ri}}$, and $\bar{v}$ are periodic because $\left.\lambda_{i}^{\prime}\right|_{\xi=0}=0$, and $\overline{\mathrm{Ro}}$ and $\bar{Q}$ are periodic because $\left.\lambda_{i}^{\prime \prime}\right|_{\xi=0}=0$ [cf. Eqs. (4), (11), and (12)]. We set $\chi_{1}=0.3 L_{x}$, in order for the secondary front to be systematically stable. We compute $B$ and $\chi_{0}$ from $f, \delta, \Phi_{0}, \mathrm{Ri}_{0}$, and $\mathrm{Ro}_{0}$.

The code of Winters et al. (2004) is pseudospectral, nonlinear, and nonhydrostatic. The set of equations solved is

$$
\begin{aligned}
& \frac{\partial \boldsymbol{v}}{\partial t}+(f \hat{\mathbf{z}}+\boldsymbol{\zeta}) \times \boldsymbol{v}+\left(f \overline{\operatorname{Ro}} u+\frac{M^{2}}{f} w\right) \hat{\mathbf{y}}=b \hat{\mathbf{z}}-\nabla p+\mathcal{D} \boldsymbol{v}, \\
& \partial_{t} b+(\boldsymbol{v} \cdot \nabla) b+M^{2} u+N^{2} w=\mathcal{D} b, \\
& \partial_{x} u+\partial_{z} w=0,
\end{aligned}
$$

where we used the identity $(\boldsymbol{v} \cdot \nabla) \boldsymbol{v}=\nabla\left(|\boldsymbol{v}|^{2} / 2\right)+\boldsymbol{\zeta} \times \boldsymbol{v}$, with $\boldsymbol{\nabla} \equiv\left(\partial_{x}, 0, \partial_{z}\right)$ and $\boldsymbol{\zeta}=\boldsymbol{\nabla} \times \boldsymbol{v}$, and merged all gradients into one dynamical pressure gradient.

The operator $\mathcal{D}$ is defined in Eq. (9). In the vertical, the simple Laplacian ensures that the number of degrees of freedom at the surface matches the four boundary conditions for the four active scalars $u, v, w$, and $b$ [for more detail, see Grisouard and Thomas (2016)]. Also, in both directions, our 2D numerical grids are fine enough to accommodate values of Laplacian diffusion coefficients that approach realistic values. Last, using only hyperdiffusion operators would have required a fine-tuning of their coefficient values for each numerical experiment. Otherwise, the dealiasing action of high-order hyperdiffusivity operators tuned for larger domains may act too aggressively on experiments that run in smaller domains.

The Laplacian coefficients are kept constant throughout, with $\nu_{h}=1 \mathrm{~m}^{2} \mathrm{~s}^{-1}$ and $\nu_{z}=2.7 \times 10^{-5} \mathrm{~m}^{2} \mathrm{~s}^{-1}$. These values are meant to be a compromise between the number of points that we can afford and the order of magnitude of typical background values for oceanic diffusivities of tracers in quiet parts of the thermocline (e.g., Ledwell et al. 1993, 1998). In other words, our values for the Laplacian coefficients are meant to be low estimates of the typical oceanic coefficients but certainly not to represent a realistic operator. Following up on this reasoning, numerical instabilities due to aliasing are blamed on $\nu_{h}$ and $\nu_{z}$ being too low. As it turns out, in all relevant cases, aliasing only happens in the horizontal direction. Therefore, for each experiment, we adjust $\nu_{3 h}$ to ensure numerical stability. The damping operator $\mathcal{D}$ does not apply to the background geostrophic flow, with negligible consequences, and the same $\mathcal{D}$ applies to both momentum and buoyancy (Prandtl number unity). We will see that the choice of $\mathcal{D}$ mostly influences the saturation and extinction stages, after we are able to draw our main conclusions.

We estimate $\alpha^{2}$ for a given initial condition with empirical guidance from the observed behavior of the fastest-growing modes. First, we assume that the horizontal half-wavelength of the instability-generated flow is on the order of $\chi_{\alpha}$, the width of the unstable, $f \bar{Q}<0$ region. We then assume that the vertical wavelength is on the order of $\delta_{\alpha}$, the depth of the unstable region. These constraints determine horizontal and vertical wavelengths $\left(k_{\alpha}, m_{\alpha}\right)=\left(\chi_{\alpha}^{-1}, 2 \delta_{\alpha}^{-1}\right) \pi$. We measure $\chi_{\alpha}$ and $\delta_{\alpha}$ for each experiment, which yields our estimate for $\alpha^{2}$, hereinafter referred to as follows:

$$
\alpha_{0}^{2}=\frac{\nu_{h} k_{\alpha}^{2}+\nu_{z} m_{\alpha}^{2}+\nu_{3 h} k_{\alpha}^{6}}{\max \left(\sigma_{0}\right)} .
$$

Table 1 shows various features of the initial conditions. In particular, it shows that $\alpha_{0}^{2}$ is usually of a few percent.

Vertically, we use a grid of 1025 points, corresponding to a resolution of $\Delta z \approx 9.8 \mathrm{~cm}$. Horizontally, domains of size XS, S, M, and L employ grids of $2^{10}, 2^{11}, 2^{12}$, and $2^{13}$ points, respectively, with $\Delta x \approx 17.09 \mathrm{~m}$ for all experiments. The time step is $\Delta t=180 \mathrm{~s}$. We initialize the simulations with random noise. Free-slip and no-buoyancy-fluctuation boundary conditions are implemented at the top and bottom, where we also taper off the frontal terms (Winters and de la Fuente 2012).

We focus on the regime contained within

$$
\begin{aligned}
& 0.9 \leq \mathrm{Ri}_{0}^{-1} \leq 1.4, \quad-1.4 \leq \mathrm{Ro}_{0} \leq-0.9, \quad \text { and } \\
& 20 \leq \Phi_{0} \leq 80 .
\end{aligned}
$$

For example, the numerical experiment characterized by $\Phi_{0}=80, \mathrm{Ri}_{0}^{-1}=1.2$, and $\mathrm{Ro}_{0}=-0.9$ is labeled N80F12R09. Table 1 lists all experiments, along with some of their specific features.

All experiments have similar life cycles, described by Thorpe and Rotunno (1989): growth, saturation, and decay. While the first two phases happen over the course of a few tens of inertial periods at the most, every experiment settles into a decay phase, which could last hundreds of inertial periods. This phase is a low-intensity regime, slowly extracting energy from the front, balanced 
TABLE 1. Some parameters of the numerical experiments. The $T_{\text {tot }}$ is in multiples of $T_{f}$.

\begin{tabular}{llcccc}
\hline \hline Experiment & Size & $T_{\text {tot }}$ & $\nu_{3 h}\left(\mathrm{~m}^{6} \mathrm{~s}^{-1}\right)$ & $\alpha_{0}^{2}(\%)$ & $\sigma_{0}(\%$ of $f)$ \\
\hline N20F09R12 & XS & 34.1 & 0 & 16 & 70 \\
N20F09R14 & XS & 14.4 & 0 & 16 & 83 \\
N20F10R12 & XS & 33.0 & 0 & 12 & 73 \\
N20F10R14 & XS & 14.0 & 0 & 12 & 85 \\
N20F11R12 & XS & 32.0 & 0 & 8.9 & 75 \\
N20F11R14 & XS & 13.8 & 0 & 9.1 & 87 \\
N20F12R09 & M & 41 & $10^{8}$ & 11 & 56 \\
N20F12R10 & S & 18.9 & 0 & 7.9 & 63 \\
N20F12R12 & XS & 15.5 & 0 & 6.6 & 77 \\
N20F12R14 & XS & 13.5 & 0 & 7.0 & 89 \\
N20F14R12 & S & 14.7 & 0 & 3.5 & 82 \\
N20F14R14 & XS & 12.9 & 0 & 4.1 & 93 \\
N50F09R12 & S & 17.0 & $7 \times 10^{7}$ & 6.2 & 70 \\
N50F09R14 & S & 14.4 & $7 \times 10^{7}$ & 4.3 & 83 \\
N50F10R10 & S & 83.4 & $7 \times 10^{7}$ & 11 & 58 \\
N50F10R12 & S & 16.6 & $7 \times 10^{7}$ & 5.1 & 73 \\
N50F10R14 & S & 14.0 & $7 \times 10^{7}$ & 3.5 & 85 \\
N50F11R09 & M & 92.3 & $8 \times 10^{8}$ & 15 & 52 \\
N50F11R10 & M & 63.9 & $4 \times 10^{8}$ & 8.5 & 60 \\
N50F11R12 & S & 16.0 & $7 \times 10^{7}$ & 4.2 & 75 \\
N50F11R14 & S & 13.7 & $7 \times 10^{7}$ & 2.9 & 87 \\
N50F12R09 & L & 31.9 & $3.5 \times 10^{10}$ & 11 & 56 \\
N50F12R10 & M & 18.9 & $8 \times 10^{8}$ & 6.5 & 63 \\
N50F12R12 & S & 15.5 & $7 \times 10^{7}$ & 3.4 & 77 \\
N50F12R14 & S & 13.5 & $2 \times 10^{8}$ & 2.5 & 89 \\
N50F14R12 & M & 14.7 & $4 \times 10^{8}$ & 2.4 & 82 \\
N80F09R12 & S & 17.0 & $2 \times 10^{8}$ & 5.1 & 70 \\
N80F09R14 & S & 14.4 & $2 \times 10^{8}$ & 2.9 & 83 \\
N80F10R10 & M & 41.8 & $2 \times 10^{9}$ & 11 & 58 \\
N80F10R12 & M & 16.6 & $4 \times 10^{8}$ & 4.3 & 73 \\
N80F10R14 & S & 14.0 & $8 \times 10^{8}$ & 2.5 & 85 \\
N80F11R09 & $\mathrm{L}$ & 92.3 & $3.5 \times 10^{10}$ & 15 & 52 \\
N80F11R10 & M & 19.8 & $8 \times 10^{9}$ & 8.2 & 60 \\
N80F11R12 & M & 16.0 & $8 \times 10^{8}$ & 3.6 & 75 \\
N80F11R14 & M & 13.7 & $4 \times 10^{9}$ & 2.2 & 87 \\
N80F12R10 & $\mathrm{L}$ & 18.9 & $3.5 \times 10^{10}$ & 6.3 & 63 \\
N80F12R12 & M & 15.5 & $2 \times 10^{9}$ & 3.1 & 77 \\
\hline & & & & &
\end{tabular}

by a slow dissipation. This regime would probably be interrupted by other processes in the restless ocean. Therefore, we run each experiment long enough for the instability to grow and saturate, but stop integration at a time $t=T_{\text {tot }}$, in an ad hoc fashion. In general, high- $\left|\mathrm{Ro}_{0}\right|$, low- $\Phi_{0}$ experiments tend to saturate faster owing to a faster breakup of the initial, negative EPV patch into smaller patches, while low- $\Phi_{0}$ and low- $\mathrm{Ro}_{0}$ experiments require longer integration periods (see Table 1$)$. As a remark, no readily available time scale, such as $\max \left(\sigma_{0}\right)$, is helpful to determine $T_{f}$.

In Table 1, constraints due to the GXR [recall Fig. 2 and Eq. (15)] are visible. For example, low- $\mathrm{Ri}_{0}$, low$\left|\mathrm{Ro}_{0}\right|$ experiments require larger domains, reflecting the divergence of $\chi_{0}$ as $\mathrm{Ri}_{0} \mathrm{Ro}_{0} \rightarrow \hat{a}$. Also notice the absence of the couples $\left(\mathrm{Ri}_{0}, \mathrm{Ro}_{0}\right)=(1.4,-0.9)$ and $(1.4,-1)$, which are in the GXR.

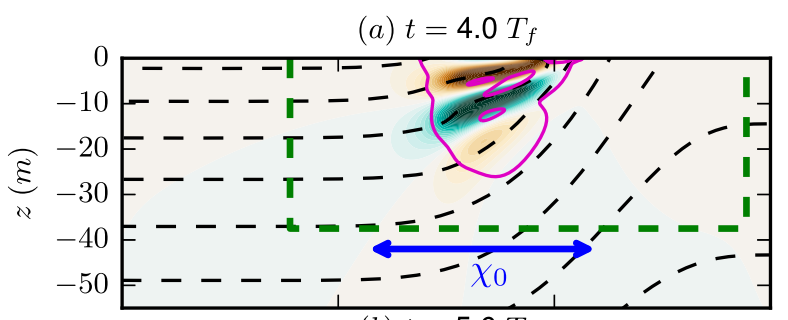

(b) $t=5.6 T_{f}$
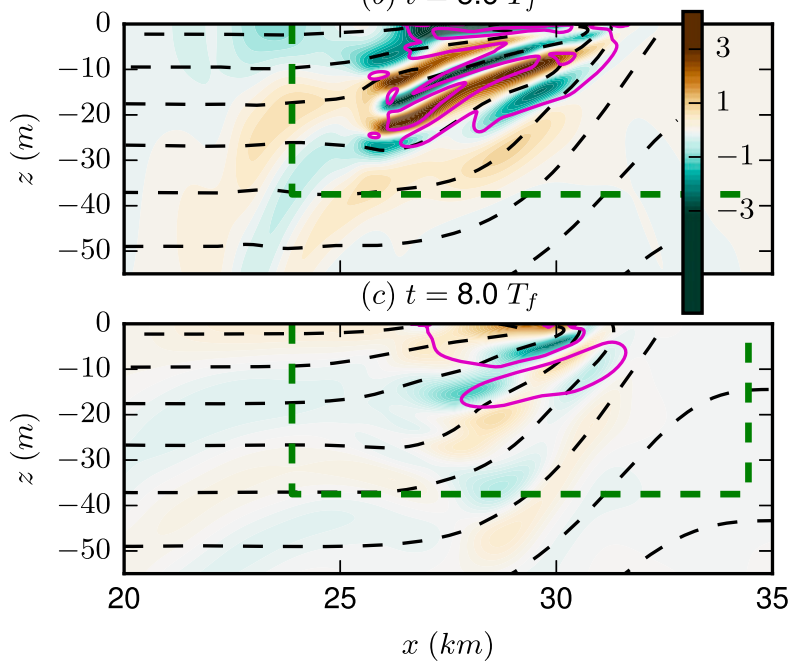

FIG. 4. Snapshots of $u\left(\mathrm{~cm} \mathrm{~s}^{-1}\right)$ for N50F12R14. As in Fig. 3, the magenta lines mark the location of the $\bar{Q}=0$ contour(s), and the green dashed rectangle marks the boundaries of the control volume for the energy budget. Black dashed lines are isopycnals.

\section{Case study: $\mathbf{R i}_{0}^{-1} \lesssim\left|\mathbf{R o}_{0}\right|$}

We now focus on the numerical experiment N50F12R14 (i.e., $\mathrm{Ri}_{0}^{-1}=1.2, \mathrm{Ro}_{0}=-1.4$, and $\Phi_{0}=50$ ). We choose this experiment because it is representative of our experimental set. In particular, it highlights some features of ISI not only as a blend between InI and SI but as an instability with genuinely original dynamics. We have $\left|\mathrm{Ro}_{0}\right|>\mathrm{Ri}_{0}^{-1}$ and, indeed, we will see (section $4 \mathrm{~b}$ ) that lateral shear production exceeds geostrophic shear production in extracting KE from the front. However, we will see that these two energetic terms only account for a significant fraction of the energy exchanges between the front and the perturbations, but not its totality.

\section{a. Time evolution}

Snapshots of the instability-induced flow are presented in Figs. 4 and 5 An animation of the flow is included in the supplemental material. During the initial growth phase (Figs. 4a and 5a), the flow forms circulation cells. Figure 5a shows the streamlines on the motion before it has grown large enough to disturb the background flow and highlights that when accommodating for the boundaries of the unstable domain, the circulation cannot happen strictly along 

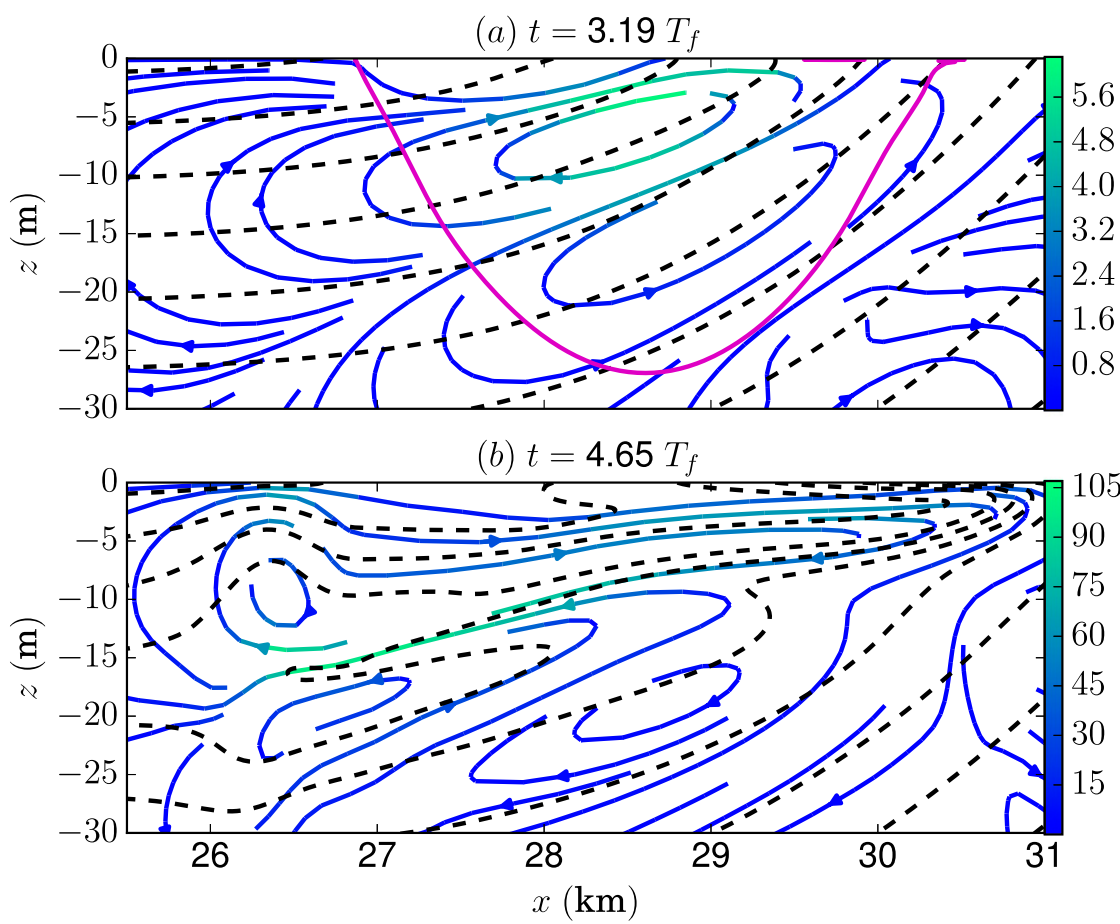

FIG. 5. Streamlines of N50F12R14 (colored lines, with the color representing $\sqrt{u^{2}+w^{2}}$; $\mathrm{mm} \mathrm{s}^{-1}$ ) and isopycnals (black dashed contours). Also in (a) are $\bar{Q}=0$ contours (magenta lines at two different stages of the instability.

isopycnals. In particular, DSS selects a scale that is fairly large compared with the size of the unstable region, which is consistent with the existing literature (cf. sections 1 and 2).

This diapycnal flow is a leading-order deviation from the linear stability analysis of boundless inviscid ISI, for which streamlines align with isopycnals. The presence of boundaries here, and DSS in general, induces this weakly diffusive (recall from Table 1 that $\alpha_{0} \approx 2.5 \%$ ) diapycnal flow that keeps growing and is bound to eventually disturb isopycnals. It is responsible for the extraction of PE. As time goes on, smaller and smaller scales develop and diffusive effects grow in importance. However, the $\mathcal{D}$ operator only plays a role in saturating the ISI. While its nature is likely to have significant quantitative effects, we claim that the existence of large PE exchanges does not depend on its specifics. The initially growing flow is a first illustration of this point, to which we will keep adding supporting evidence over the course of this article.

At $t=4 T_{f}$ (Fig. 4a), contours of $\bar{Q}=0$ start being deformed by the instability. As the instability grows, the $\bar{Q}=0$ contours break down into smaller and smaller filament-like regions (Figs. $4 \mathrm{~b}$ and $5 \mathrm{~b}$ ), reaching saturation. As noticed by Thorpe and Rotunno (1989), the creation of these small scales stops the growth of the instability. The initial conditions, which allowed the jet-scale ISI to develop, are no longer present, and the ISI chokes. Note that the remaining pockets of negative EPV are all susceptible to ISI individually. However, they would need some time to grow and diabatic effects may hinder their growth. Moreover, no stationary state is achieved in N50F12R14 as in most of the numerical experiments: pockets of negative EPV keep evolving and sometimes merge, causing ISI to reappear episodically.

In the later stages of the ISI growth, streamlines and isopycnals eventually align (Fig. 5b), as in pure SI. However, this happens only in the core of the unstable zone. Outside of this core (see, e.g., Fig. 5b), around $x=27 \mathrm{~km}, z=-15 \mathrm{~m}$, density overturns. Overturns eventually disappear, although N50F12R14 does not exhibit any gravitational instabilities at these locations. A few possible explanations can be provided as to why we observe no gravitational instability. First, studies on convectively unstable internal waves (e.g., Winters and Riley 1992; Winters and D'Asaro 1994; Lelong and Dunkerton 1998) suggest that the fastest modes of convective instability are vortex rolls with axes that largely align with the along-propagation direction (the $x$ direction here). Because our two-dimensional simulations do not allow this three-dimensional mode of motion to exist, convective instability may take too long to develop here in a context of a rapidly evolving flow. Another reason could be that at this stage of the 
instability, dissipation is the dominant energetic process and prevents the rapid growth of the instability.

Figure $4 \mathrm{~b}$ and the animation in the supplemental material show that when saturating, internal waves radiate from the anticyclonic flank of the unstable region, as described qualitatively by Grisouard et al. (2016). However, we will see that this represents a very small fraction of the energy extracted from the front.

After some time (Fig. 4c), the dynamics enter an extinction phase during which ISI remains active, although less intensely so. Like Thorpe and Rotunno (1989), none of our simulations reach a state in which ISI is completely extinguished.

\section{b. Energy budget}

Derived from Eqs. (18), the budgets of energy densities are

$\partial_{t} \mathcal{K}+\nabla \cdot \mathbf{F}_{\mathcal{K}}-b w+\overbrace{\frac{M^{2}}{f} v w}^{\mathrm{GSP}}+\overbrace{f \overline{\mathrm{Ro}} u v}^{\mathrm{LSP}}=-\varepsilon_{\mathcal{K}}, \quad$ and

$\partial_{t} \mathcal{P}+\nabla \cdot \mathbf{F}_{\mathcal{P}}+b w \underbrace{-\frac{b^{2} \boldsymbol{v} \cdot \nabla N^{2}}{2 N^{4}}}_{\mathrm{HABP}}+\underbrace{\frac{M^{2}}{N^{2}} u b}_{\mathrm{GBP}}=-\varepsilon_{\mathcal{P}}$,

where $\mathcal{K}=|\boldsymbol{v}|^{2} / 2$ is the kinetic energy density, and $\mathcal{P}=b^{2} / 2 N^{2}$ is a definition of the potential energy density, suitable for our purposes. The energy fluxes $\mathbf{F}_{\mathcal{K}}$ and $\mathbf{F}_{\mathcal{P}}$ include those due to pressure work, advection, and diffusion. The LSP and GSP are defined in Eqs. (2) and (3). Two buoyancy production terms appear in Eq. (20b):

$\mathrm{HABP}=-\frac{b^{2} \boldsymbol{v} \cdot \nabla N^{2}}{2 N^{4}} \quad$ and $\quad \mathrm{GBP}=\frac{M^{2}}{N^{2}} u b$,

which stand for horizontal ageostrophic and geostrophic buoyancy production, respectively.

Finally, the dissipation terms are

$\varepsilon_{\mathcal{K}}=\left(\nu_{h} \boldsymbol{v}_{, x}+\nu_{3 h} \boldsymbol{v}_{, 5 x}\right) \cdot \boldsymbol{v}_{, x}+\nu_{z}\left(\boldsymbol{v}_{, z}\right)^{2}, \quad$ and

$\varepsilon_{\mathcal{P}}=\left(\nu_{h} b_{, x}+\nu_{3 h} b_{, 5 x}\right)\left(\frac{b}{N^{2}}\right)_{, x}+\nu_{z} b_{, z}\left(\frac{b}{N^{2}}\right)_{, z}$,

where $(\cdot)_{, n x}=\partial_{x}^{n}(\cdot)$.

Recall that for pure InI and SI, LSP and GSP are respectively the sole source terms, while isopycnals remain unperturbed, and HABP and GBP are identically zero. Figure 6 shows snapshots, taken at $t=$ $4.65 T_{f}$, of LSP, GSP, and GBP. The HABP is comparatively negligible, here and in all of our experiments. Figure $6 c$ demonstrates that GBP acts as an energy source for the perturbations and that at least in

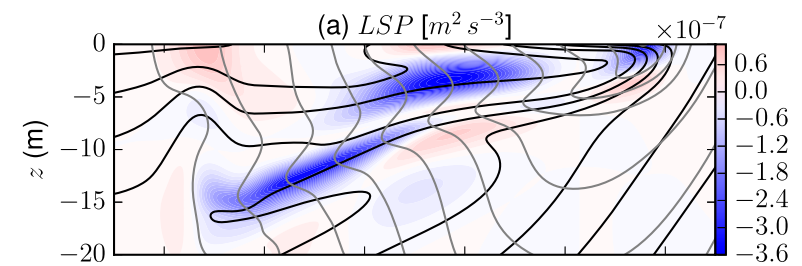

(b) $G S P\left[m^{2} s^{-3}\right]$

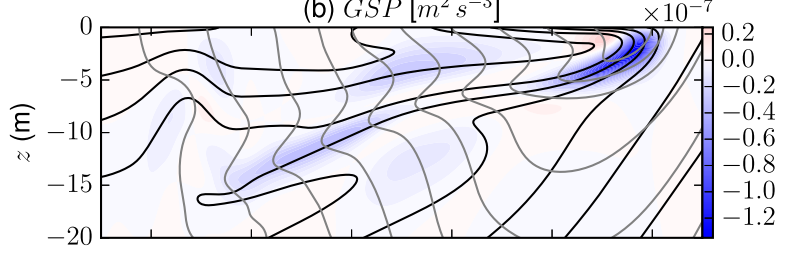

(c) $G B P\left[m^{2} s^{-3}\right]$

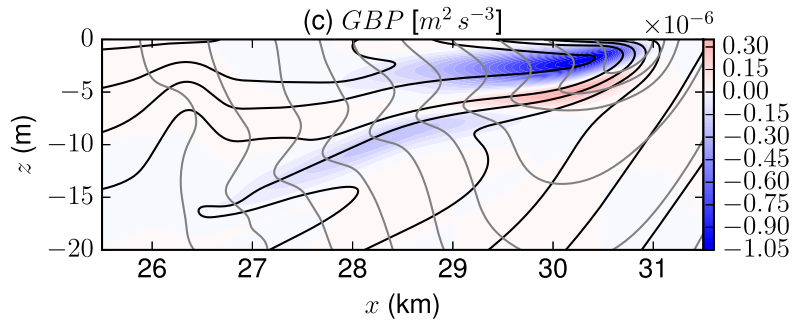

FIG. 6. Snapshots of the three most important sources of energy for the perturbations in N50F12R14 (shading), isopycnals (black contours), and $v+\bar{v}$ (gray contours), at time $t=4.65 T_{f}$. Blue indicates a transfer of energy from the background to the perturbations.

some locations and at that instant, it is actually a larger source of energy for the perturbations than LSP and GSP.

To quantify these statements, we integrate Eqs. (20) over the control volume $\mathcal{V}$ that is visible in Figs. 3 and 4 inside a dashed, rectangular contour. It is defined by

$$
L_{x}-2 \chi_{0} \leq x \leq L_{x}-\chi_{0} / 10 \text { and }-L_{z} / 3 \leq z \leq 0 .
$$

This definition ensures that for the present experiment as well as all those listed in Table 1, all ISI-induced perturbations are contained within the control volume. We add Eqs. (20) and integrate over $\mathcal{K}$, with $\langle\cdot\rangle=\iint_{\mathcal{V}} \cdot d x d z$, in order to form a mechanical energy budget for $\mathcal{M}=\mathcal{K}+\mathcal{P}$. For normalization purposes, we also define a reference value for the energy as the approximation of $\left\langle\bar{v}^{2}\right\rangle / 2$ if the basic flow only comprised the unstable front in a semi-infinite domain in both directions [cf. Eqs. (12) and (17)]:

$$
\begin{aligned}
\left\langle\mathcal{K}_{0}\right\rangle & =\frac{1}{2} \frac{B^{2} \delta^{2}}{f^{2} \chi_{0}^{2}} \int_{-\infty}^{0} e^{2 z / \delta} d z \int_{-\infty}^{L_{x}}\left[\lambda_{i}^{\prime}\left(\frac{L_{x}-x}{\chi_{0}}\right)\right]^{2} d x \\
& =\frac{\Gamma(2 / 3)}{2^{8 / 3}} \frac{B^{2} \delta^{3}}{f^{2} \chi_{0}},
\end{aligned}
$$

with $\Gamma(\xi)=\int_{0}^{\infty} x^{\xi-1} e^{-x} d x$ the gamma function. We normalize the integrals of the power terms in Eqs. (20) by 


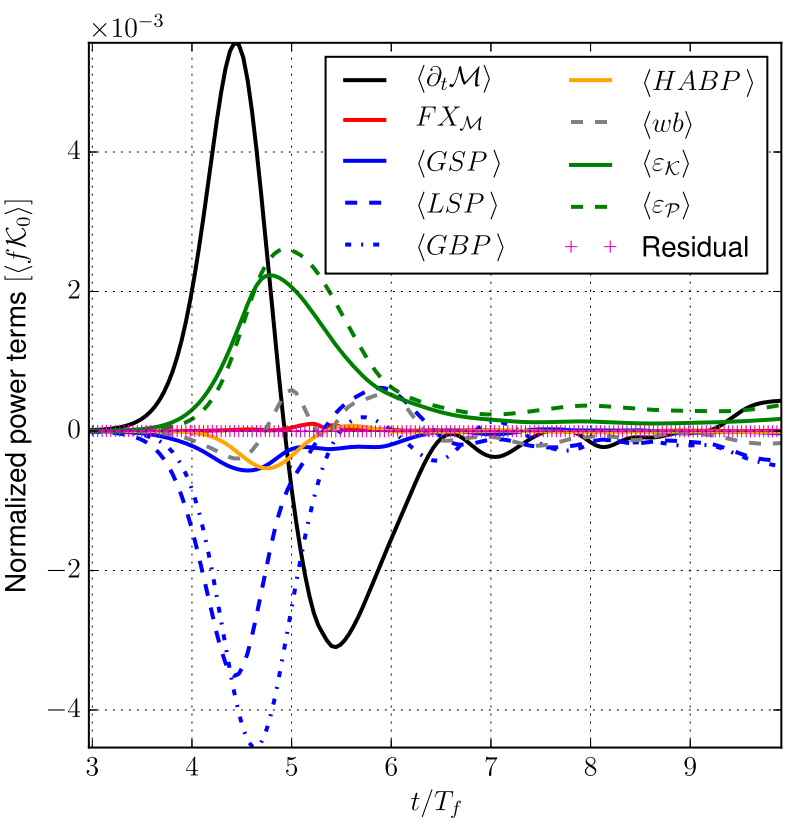

FIG. 7. Time series of the normalized integrals of the terms involved in the mechanical energy budgets [Eq. (20)], for $\mathrm{N} 50 \mathrm{~F} 12 \mathrm{R} 14$. The buoyancy production term $\langle w b\rangle$ is not included in the residual.

$\left\langle f \mathcal{K}_{0}\right\rangle$. Figure 7 shows time series of these terms. The internal wave energy flux, $\mathrm{FX}_{\mathcal{M}}=\oint_{\partial \mathcal{V}} \mathbf{F}_{\{\mathcal{K}+\mathcal{P}\}} \cdot \hat{\mathbf{n}} d \ell$, with $\hat{\mathbf{n}}$ the outward normal to $\mathcal{V}$, represents a very small fraction of the energy lost by the perturbations, most of it being dissipated inside $\mathcal{V}$. The buoyancy production term $\langle w b\rangle$ is also small, showing that within the mechanical energy budget, KE to PE exchanges are small.

Integrating $\langle\mathrm{LSP}\rangle,\langle\mathrm{GSP}\rangle,\langle\mathrm{GBP}\rangle$, and $\langle\mathrm{HABP}\rangle$ over time and normalizing by their sum,

$\theta_{X}=\frac{1}{\varsigma} \int_{0}^{T_{\text {tot }}}\langle X\rangle d t, \quad$ with $\quad \varsigma=\sum_{X} \int_{0}^{T_{\text {tot }}}\langle X\rangle d t$,

where $X=$ GBP, LSP, GSP, or HABP, shows that $\theta_{\mathrm{GBP}} \approx 53 \%$ (i.e., that GBP represents $53 \%$ of the total energy extracted from the front), $\theta_{\mathrm{LSP}} \approx 34 \%$, $\theta_{\mathrm{GSP}} \approx 9 \%$, and $\theta_{\mathrm{HABP}} \approx 4 \%$. Therefore, the contributions from the PE budget are important to the total energy budget of the ISI. This is a significant departure from the narrative that InI and/or SI only extract $\mathrm{KE}$ from fronts and confirms the intuitions of Colin de Verdière (2012) and Bachman and Taylor (2014).

Finally, note that dissipation always lags extraction. This is another indication that the energy exchanges are not primarily controlled by the operator $\mathcal{D}$, though a quantitative influence may exist.

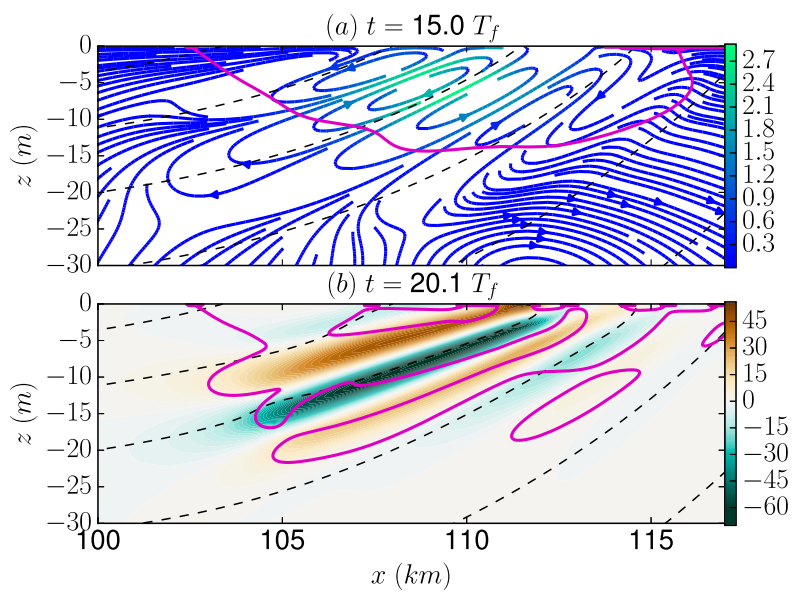

FIG. 8. Snapshots of N50F12R09, for (a) streamlines whose color is indexed by $\sqrt{u^{2}+w^{2}}\left(\mathrm{~mm} \mathrm{~s}^{-1}\right)$ and (b) $u\left(\mathrm{~mm} \mathrm{~s}^{-1}\right)$. Magenta contours mark the $\bar{Q}=0$ contours.

\section{Toward the limiting cases}

N50F12R14 exhibits more energy extraction from the front due to geostrophic buoyancy production [GBP; cf. Eq. (21)] over lateral and geostrophic shear productions [LSP and GSP, respectively; cf. Eqs. (2) and (3)], which highlights the importance of the PE budget when ISI is a blend of InI and SI. To connect the previous finding with the bulk of the existing literature on SI and InI, we now use two experiments, which approach these limiting cases. Even though GBP will remain a significant term throughout this study, we will draw inspiration from the classification of Thomas et al. (2013), in qualifying experiments as InI-like if $\theta_{\text {LSP }}>$ $\theta_{\mathrm{GSP}}$ [cf. Eq. (25)], and SI-like otherwise.

N50F12R09 is closer to a pure SI case, with $\left|\mathrm{Ro}_{0}\right|<\mathrm{Ri}_{0}^{-1}$. Figure 8 shows snapshots of the experiment in its growing (Fig. 8a) and saturation (Fig. 8b) phases. Closer to what one would expect from ISI in an infinite domain, the flow is mostly isopycnal and remains so, even in the saturation phase and after (not shown). In Fig. 8a, and as opposed to Fig. 5a, it appears that isopycnal flow is favored by the aspect ratio of the unstable region, which is wider when the isopycnals are steep:

$$
\gamma=\frac{\chi_{\alpha}}{\delta_{\alpha}} \frac{M_{1}^{2}}{N_{1}^{2}} \approx 3
$$

with $\chi_{\alpha} / \delta_{\alpha}$ the aspect ratio of the unstable region as defined in section $3 \mathrm{~b}$, and $M_{1}^{2} / N_{1}^{2}$ the isopycnal slope at the location where $f \bar{Q}$ is minimum. In comparison, N50F12R14 was characterized by $\gamma \approx 0.9$; that is, the front was much narrower, which under DSS seems to have been an additional geometric constraint on the initial circulation cells. 


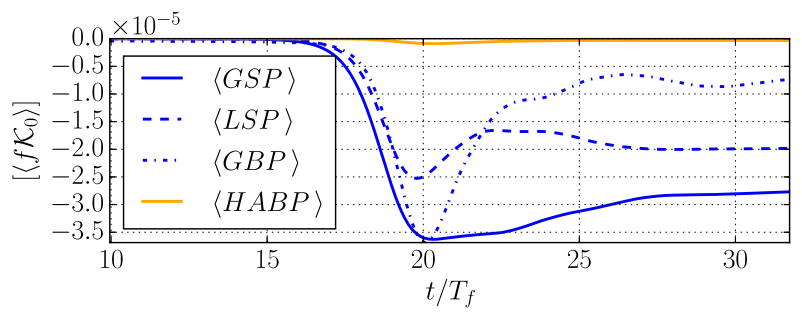

FIG. 9. Time series of the normalized integrals of the terms, extracting energy from the geostrophic front, for N50F12R09.

Nonetheless, GBP is again a significant source of energy for the perturbations, if not as important as in N50F12R14. Figure 9 shows time series of the terms, which extract energy from the geostrophic flow. We find that GSP is indeed the dominant extraction term, consistent with the SI-like character of our initial conditions, but GBP and LSP are also significant sources of energy for the perturbations. After time integrating these time series, we find $\theta_{\mathrm{GSP}} \approx$ $48 \%, \theta_{\mathrm{GBP}} \approx 21 \%$, and $\theta_{\mathrm{LSP}} \approx 30 \%$. However, after a first initial phase during which GSP and GBP extract as much energy from the front as each other, the instability enters a slower phase in which GBP becomes less important than both other terms. Therefore, we can expect $\theta_{\mathrm{GSP}}$ to increase with time and $\theta_{\mathrm{GBP}}$ to decrease.

On the other end of our spectrum, with N50F09R14, $\left|\mathrm{Ro}_{0}\right|>\mathrm{Ri}_{0}^{-1}$ and we approach the pure-InI case. The normalized aspect ratio is now $\gamma \approx 0.7$. Figure $10 \mathrm{a}$ shows a snapshot of the experiment in its growing phase, while Fig. 10b displays time series of the relevant power terms. As expected, GSP is again weak $\left(\theta_{\mathrm{GSP}} \approx 9 \%\right)$ and LSP is slightly larger than GBP, with $\theta_{\mathrm{LSP}} \approx 49 \%$ and $\theta_{\mathrm{GBP}} \approx 41 \%$.

\section{Parameter exploration}

\section{a. On the relative importance of PE exchanges}

In the previous sections, we have illustrated typical behaviors, found in our set of numerical experiments. They illustrated the main message of the present article, namely, that inertial-symmetric instabilities involve important, and perhaps dominant, PE exchanges between the geostrophic flow and the ageostrophic perturbations. Let us lend further support to these arguments with the full set of 37 experiments listed in Table 1.

Our first task is to graphically represent the values of three scalars, namely, $\theta_{\mathrm{GBP}}, \theta_{\mathrm{LSP}}$, and $\theta_{\mathrm{GSP}}$, each of which being described in a three-dimensional parameter space. Note that, consistently with the three case studies presented earlier, $\theta_{\mathrm{HABP}}$ never exceeds $6 \%$, and will be ignored. For each remaining scalar, we use three scatterplots, each of which collapses the data along one independent parameter. However, we cannot use $\mathrm{Ri}_{0}, \mathrm{Ro}_{0}$,
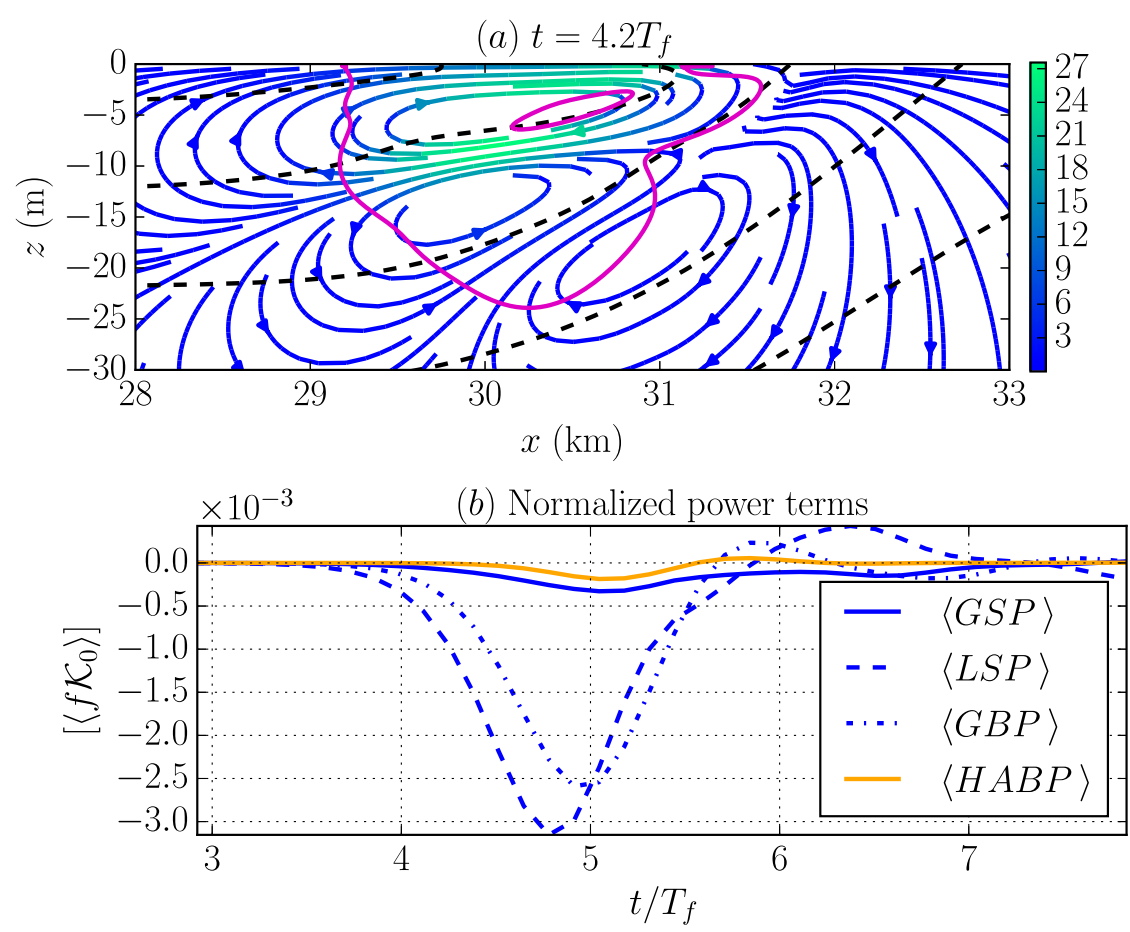

FIG. 10. Evolution of the instability in N50F09R14. (a) Streamlines whose color is indexed by $\sqrt{u^{2}+w^{2}}\left(\mathrm{~mm} \mathrm{~s}^{-1}\right)$, as in Fig. 5. The magenta contour marks the $\bar{Q}=0$ contour. (b) Time series of the normalized integrals of the terms, extracting energy from the geostrophic front. 

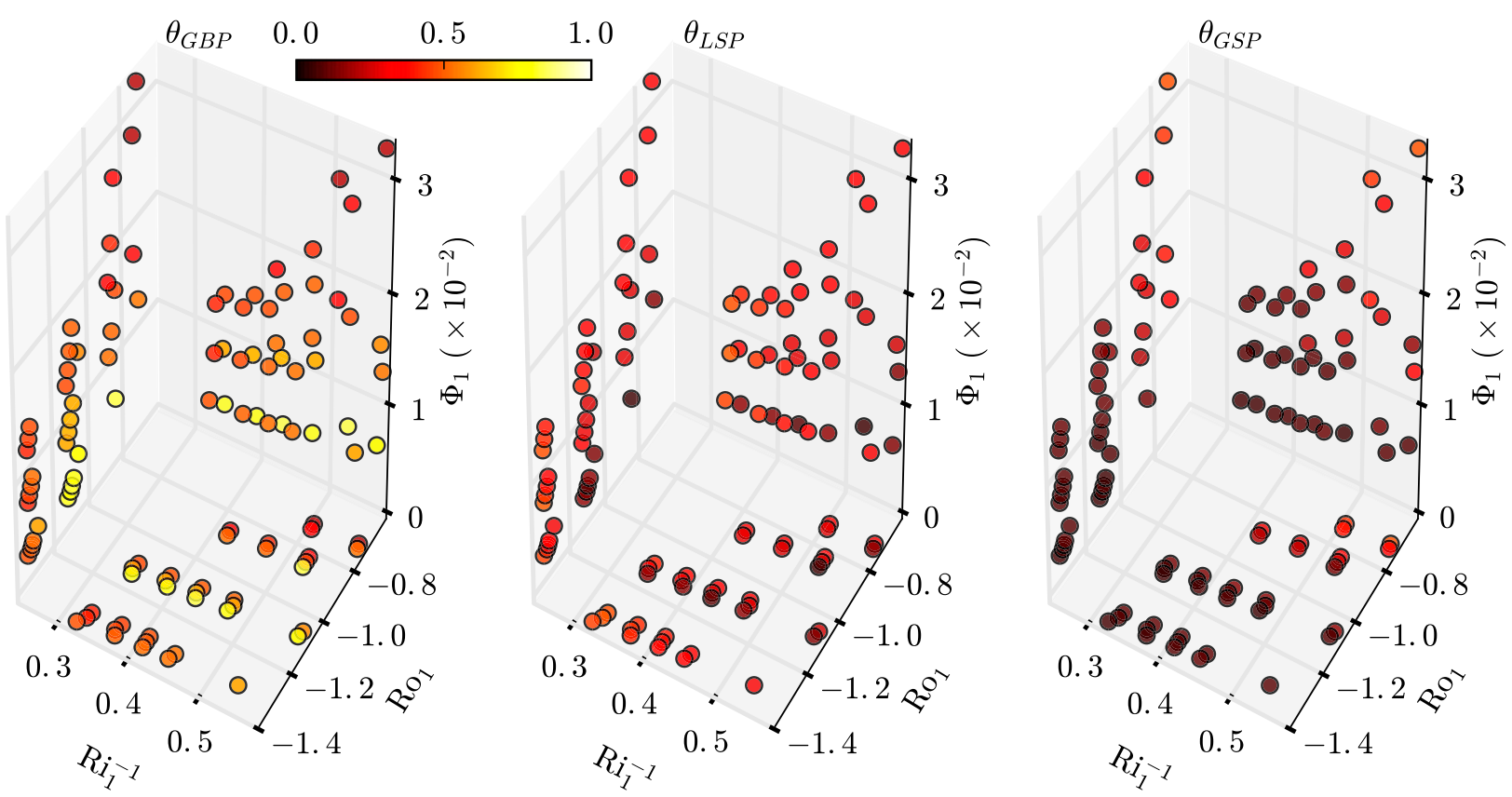

FIG. 11. Scatterplots of (left) $\theta_{\mathrm{GBP}}$, (center) $\theta_{\mathrm{LSP}}$, and (right) $\theta_{\mathrm{GSP}}$, each dot representing a numerical experiment and its color the magnitude of the corresponding term. Each quantity is collapsed onto each one of the three axes of the parameter space (i.e., $\mathrm{Ri}_{1}^{-1}$, $\mathrm{Ro}_{1}$ and $\Phi_{1}$ ). Therefore, each quantity is displayed three times, once on each face of a given cube.

and $\Phi_{0}$ as axes of our three-dimensional representation, since data points would collapse onto each other and the results would be impossible to read. Instead, and somewhat arbitrarily, we redefine our basis by first locating the point in the initial conditions where $f \bar{Q}$ is minimum. At that location, we evaluate the Richardson number, the Rossby number, and the inverse Prandtl ratio, which yields $\mathrm{Ri}_{1}, \mathrm{Ro}_{1}$, and $\Phi_{1}$, respectively (cf. section 3a).

Figure 11 presents these nine scatterplots. Each face of a cube contains 37 entries, corresponding to their coordinates in either $\left(\mathrm{Ro}_{1}, \Phi_{1}\right),\left(\Phi_{1}, \mathrm{Ri}_{1}^{-1}\right)$, or $\left(\mathrm{Ri}_{1}^{-1}, \mathrm{Ro}_{1}\right)$ spaces. Each entry is color coded by the value of $\theta_{\mathrm{GBP}}$, etc., and therefore, the sum of the values displayed at one location across the three panels yields $1-\theta_{\mathrm{HABP}} \approx 1$. To better distinguish entries on the $\left(\mathrm{Ri}_{1}^{-1}, \mathrm{Ro}_{1}\right)$ plots, we shift $\mathrm{Ro}_{1}$ by +0.02 for entries for which $\Phi_{0}=80$, and by -0.02 when $\Phi_{0}=20$.

With Fig. 11, we confirm that, indeed, ISI can extract a significant amount of PE from fronts. A quick comparison of the color distribution across panels indeed reveals the predominance of GBP over the other two terms, often representing at least $50 \%$ of the exchanges, sometimes less, but often more. On the other hand, GSP is the weakest term in the vast majority of the experiments, rarely accounting for more than $25 \%$ of the exchanges. Finally, LSP often takes intermediate values. Therefore, from an energetic point of view, our experiments tend to be InI-like ISI.
Our main message is that in our set of experiments, $\mathrm{PE}$ exchanges are significant and that we expect this finding to extend to the ocean. We now try to interpret additional information, present in Fig. 11. However, because these interpretations will not be backed by any physical model or mechanism, the arguments laid out in the next two subsections do not carry the same weight as our main message.

\section{b. On the weakness of GSP}

The reason why GSP is usually weak is that in most of our experiments, the instability is triggered because $|\overline{\mathrm{Ro}}|$ is high rather than because $\overline{\mathrm{Ri}}$ is low. Therefore, wherever the instability starts, one could say that ISI is more inertial than symmetric. We already proposed two physical explanations when we described our setup design in section $3 \mathrm{a}$. The first is that 1 ) in our experiments, vorticity is slightly cyclonic where the Richardson is at its lowest, while thermal wind shear helps to destabilize the jet where the Rossby number is still strongly anticyclonic (Fig. 1). The second explanation is 2) the existence of a GXR; that is, the geometry of our front excludes combinations of low $\left|\mathrm{Ro}_{0}\right|$ and low $\mathrm{Ri}_{0}$ numbers, making SI-like experiments difficult to set up.

Two more explanations point at limitations of the numerical setup. 3) Fronts with low $\mathrm{Ri}_{0}$ and low $\left|\mathrm{Ro}_{0}\right|$ approach the divergence at the edge of the GXR and therefore tend to have wider aspect ratios [cf. Eq. (A5)], 


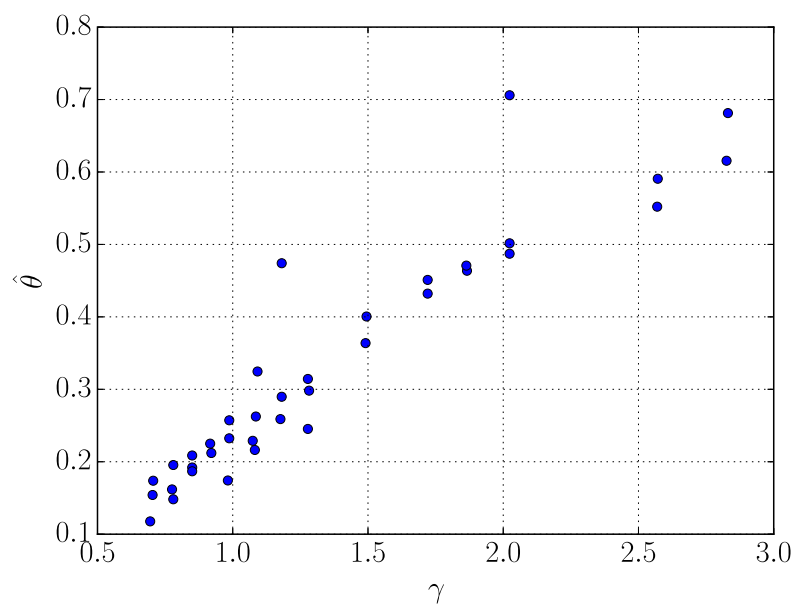

FIG. 12. Fraction of the KE extracted from the front by $\langle\mathrm{GSP}\rangle$, as a function of the normalized aspect ratio $\gamma$. Each dot corresponds to a numerical experiment.

requiring larger domains, which is why we decided not to run N80F12R09. And 4) numerical stability of experiments with low $\mathrm{Ri}_{0}$ and low $\left|\mathrm{Ro}_{0}\right|$ also happen to require much higher hyperviscosity coefficients (cf. Table 1), which is why we decided not to run N80F12R14 and N80F14R12. Explanations 3 and 4 are exacerbated when $\Phi_{1}$ is high, which also happens to be the regime in which GSP is most important, according to Fig. 11. Therefore, our decision to not run these experiments probably eliminated a handful of SI-like experiments from our set of experiments.

Based on visual comparisons of Figs. 5, 8, and 10, we proposed in section 5 that an SI-like ISI requires a wide front (i.e., a front of aspect ratio that is shallower than the typical isopycnal slope) and used $\gamma$ [cf. Eq. (26)] to quantify this statement. We follow up on this proposal, keeping in mind that it is not backed by any rigorously derived physical model. To know whether an experiment is more SI- than InI-like, we only need to compare GSP and LSP, for which we define

$$
\hat{\theta}=\theta_{\mathrm{GSP}} /\left(\theta_{\mathrm{GSP}}+\theta_{\mathrm{LSP}}\right) .
$$

Our proposal is therefore that $\hat{\theta} \rightarrow 1$ when $\gamma \rightarrow \infty$ and that $\hat{\theta} \rightarrow 0$ when $\gamma \rightarrow 0$. Figure 12 broadly agrees with this statement, except for two outliers, at $\gamma \approx 1.2$ $(\hat{\theta} \approx 0.47)$ and $\gamma \approx 2.0(\hat{\theta} \approx 0.7)$. They correspond to N20F11R12 and N20F12R10, two experiments for which $\theta_{\mathrm{GBP}} \approx 90 \%$ and for which both GSP and LSP are physically relatively meaningless. These are reminders that considering only LSP and GSP may yield little physical understanding. For example, it turns out that $\gamma$ does not depend much on any measure of $\Phi$, and neither does $\hat{\theta}$. On the other hand, $\theta_{\mathrm{GSP}}$ is greater when
$\Phi_{1}$ is high, because it usually also corresponds to lower values of $\theta_{\mathrm{GBP}}$ (Fig. 11). Also, using $\gamma$ as abscissa for $\theta_{\mathrm{GSP}}, \theta_{\mathrm{LSP}}$, and $\theta_{\mathrm{GBP}}$ yields very poor results, because $\theta_{\mathrm{GSP}}$ does not correlate at all with $\gamma$. Another reminder that without a physical model, interpretation of these numbers can be of only limited value.

\section{c. The parameter space: Two- or three-dimensional?}

We just mentioned that GSP is more important in high-stratification or low-latitude experiments. It then seems that $\mathrm{Ro}_{0}$ and $\mathrm{Ri}_{0}$ are not the sole parameters that determine whether an experiment will be more InI-like than SI-like but that at least a third parameter is involved, as predicted in section $3 \mathrm{a}$. That $\mathrm{Ri}_{1}$ and $\mathrm{Ro}_{1}$ are not sufficient can be easily seen in the $\left(\mathrm{Ri}_{1}^{-1}, \mathrm{Ro}_{1}\right)$ scatterplots. In several cases, exemplified when, for example, $\mathrm{Ro}_{1} \approx-1.2$ (recall that in Fig. 11, these values are artificially shifted), identical couples of values yield very different numbers for $\theta_{\mathrm{GBP}}, \theta_{\mathrm{LSP}}$, and $\theta_{\mathrm{GSP}}$. In fact, the Richardson number appears to be a poor predictor for the various $\theta_{X}$.

On the other hand, $\Phi_{1}$ and $\left|\mathrm{Ro}_{1}\right|$ appear to be better predictors of our results. Indeed, the $\left(\mathrm{Ro}_{1}, \Phi_{1}\right)$ panels show that higher values for $\Phi_{1}$ and $\left|\mathrm{Ro}_{1}\right|$ correspond to higher values of $\theta_{\mathrm{GBP}}$ and lower values of $\theta_{\mathrm{LSP}}$. The $\theta_{\mathrm{GSP}}$ takes higher values for high $\Phi_{1}$ and low $\left|\mathrm{Ro}_{1}\right|$, independently of $\mathrm{Ri}_{1}$. The last statement can appear surprising at first, given that both $\mathrm{Ri}_{1}$ and GSP are associated with SI. It also seems to rule out $\mathrm{Ri}_{1}$ as a meaningful nondimensional parameter, bringing the dimensionality of the parameter space from three back to two. This may be interpreted in the light of our previous comments on how SI-like instabilities are underrepresented in our dataset, for reasons both physical and technical. Thus, it is not surprising that our experiments are well described by the couple of parameters that characterize InI-like experiments. Moreover, if SI-like instability is found at low $\left|\mathrm{Ro}_{1}\right|$, then according to Fig. 2, $\mathrm{Ri}_{1}$ can only take a limited range of values. Therefore, our dataset does not rule out the possibility that GSP could be important when $\mathrm{Ri}_{1}^{-1}$ is significantly larger than $\left|\mathrm{Ro}_{1}\right|$, regardless of the value of $\mathrm{Ro}_{1}$. In conclusion, our results are too limited to draw any conclusion on the number of dimensions of our parameter space.

\section{d. Diffusive effects}

We mentioned in sections 1 and 2 the possibility that diffusive effects may have an influence on the quantitative outcome of this study. Recall that according to linear stability analysis, scale selection depends on the diffusivity coefficients of momentum and buoyancy, even when diffusive effects are too weak to noticeably impact the time scale of the instability in its linear growth phase. 
However, we can take advantage of the fact that we do not try to control the nondimensional intensity of diffusive effects. This is reflected in Table 1, which shows that the parameter $\alpha_{0}^{2}$ covers a relatively wide range of (small) values. The explanation is that while the combined variations of $\mathrm{Ri}_{0}, \mathrm{Ro}_{0}$, and $\Phi_{0}$ create fronts of widely different sizes, we keep the values of $\nu_{z}$ and $\nu_{h}$ [cf. Eq. (9)] constant across experiments. Larger domains do tend to be associated with larger values of $\nu_{3 h}$, but the hyperdiffusion operator mainly acts on the smaller scales, created after the linear growth phase. Therefore, it is mostly the harmonic operator coefficients $\nu_{z}$ and $\nu_{h}$ that set the value of $\alpha_{0}^{2}$. Nonetheless, our various attempts to recast the parameter space along different axes including $\alpha_{0}^{2}$ (say, $\mathrm{Ro}_{1}$, $\mathrm{Ri}_{1}$, and $\alpha_{0}^{2}$ ), and plotting the corresponding equivalent to Fig. 11, did not yield any noticeable pattern (not shown).

We also decided to run an experiment, identical to N50F12R14 (described in section 4), except for the diffusion operator. In this alternative experiment, we use

$$
\mathcal{D}^{\prime}=\nu_{3 h} \partial_{x}^{6}+\nu_{3 z} \partial_{z}^{6},
$$

with $\nu_{3 h}=10^{10} \mathrm{~m}^{6} \mathrm{~s}^{-1}$ and $\nu_{3 z}=10^{-9} \mathrm{~m}^{6} \mathrm{~s}^{-1}$. As a comparison, combining these values with the same $k_{\alpha}$ and $m_{\alpha}$ as for the original N50F12R14 [recall Eq. (9)] yields $\alpha_{0}^{2} \approx 5 \times 10^{-5}$, three orders of magnitude smaller than that of N50F12R14. Consistently with the predictions of linear stability analysis, however, the scale that is selected is much shorter than the one in N50F12R14 in both $x$ and $z$ directions (not shown). Consequently, the effective value of $\alpha_{0}^{2}$ must be larger, although we did not attempt to compute it. Remarkably, however, the final numbers that we deem relevant to the discussion are similar to those of N50F12R14: $\theta_{\mathrm{GBP}}=46 \%, \theta_{\mathrm{LSP}}=39 \%$, and $\theta_{\mathrm{GSP}}=9 \%$. One should not overinterpret how similar these values are to those obtained in section $4 \mathrm{~b}(53 \%, 34 \%$, and $9 \%$, respectively). Some coincidence cannot be excluded.

By themselves, the two results described above do not rule out the possibility that diffusive effects may impact energy exchanges. A more satisfactory strategy would be to run a parallel set of 37 experiments, only with lower (hyper)diffusivity coefficients, which would necessitate a finer resolution. In light of the numerical cost to run and process these experiments, and the lack of promise from our preliminary attempts to detect a robust effect, we decided against it. It may be worthwhile to investigate this issue in a future study.

\section{Conclusions and perspectives}

We demonstrated numerically that inertial-symmetric instability (ISI) of surface oceanic density fronts with finite width and depth induce exchanges of potential energy (PE) with the geostrophic flow and that these exchanges can be as important as those of kinetic energy (KE). The geometric interpretation is rather simple: in our simulations, viscid ISI selects a mode that grows within the $f \bar{Q}<0$ region, a process we referred to as diffusive scale selection (DSS). Even though ISI tends to enforce along-isopycnal flow within the unstable region, the finite size of the former necessarily implies diapycnal recirculations around it. In sections 4 and 5, we presented two such cases, which we called InI-like ISI, but which we could have called baroclinic inertial instabilities. They are instabilities that arise because of a large negative $\overline{\mathrm{Ro}}$ in the presence of a lateral density gradient and which gain energy from the fronts' KE as well as PE. In section 5, we also presented a case with large $\Phi_{1}$, small $|\overline{\mathrm{Ro}}|$, and small $\overline{\mathrm{Ri}}$. In this case, the front was wide enough for the circulation cells to align more with isopycnals, and the PE exchanges were reduced. It approached the predictions of standard linear stability theory for SI-like ISI such as that laid out by Thomas et al. (2013), that is, with GSP dominating LSP (and GBP).

Systematically, once the instability has reached a critical amplitude, the ageostrophic flow irreversibly displaces isopycnals. In our numerical experiments, GBP systematically represents a sink of PE for the geostrophic flow. This result is somewhat surprising because ISI can be considered a blend of inertial and symmetric instabilities [in the sense of Thomas et al. (2013)] and because, taken individually, these two instabilities mostly impact the KE of fronts. To our knowledge, the existing body of literature on loss of PE of geostrophic flows focuses on two processes: mixed layer instabilities and strain-induced frontogenesis (McWilliams 2016, and references therein). These two processes convert some PE of the geostrophic flow into $\mathrm{KE}$, creating flows that tend to flatten isopycnals. Here, GBP converts PE from the geostrophic front into PE of ageostrophic perturbations, which eventually feed energy dissipation. Conversion into KE is not as important as dissipation, and its sign is not constant (Fig. 7).

In designing our experiments, we had to introduce a third nondimensional parameter. For convenience, we chose $\Phi$, a measure of stratification versus Coriolis frequency, although other choices could have been considered. Figure 11 reminds us that $\Phi$ is actually a very important parameter. This figure hints at a distinction between high- and low-stratification ISI, but perhaps more interestingly, low- and high-latitude ISI, the former being characterized by low PE exchanges, and vice versa. On the other hand the Richardson number has the least predictive potential (sections $6 \mathrm{~b}$ and $6 \mathrm{c}$ ). To parameterize the energetic impact of ISI on geostrophic flows, we need to better understand whether the 
parameter space is two- or three-dimensional, as well as the predictive power of these parameters.

Our results rely on $\Phi$ being moderate. In cases where $\Phi$ is very large, the relative intensity of PE exchanges seems to diminish in our experiments. In the extreme case of being very close to the equator $(\Phi \rightarrow \infty)$, Colin de Verdière and Schopp (1994) and Colin de Verdière (2012) predict that nontraditional effects would play a significant role, introducing the need for more nondimensional parameters. In the other extreme case where $\Phi$ would be closer to unity (i.e., away from the equator and very weak stratification), Colin de Verdière (2012) also predicts that nontraditional effects would play a significant role.

Not all couples of Richardson and Rossby numbers are allowed in our setup, which may apply to actual oceanic fronts. Indeed, Eq. (15) and Fig. 2 illustrate that as the Rossby number decreases in magnitude, the Richardson number cannot take arbitrarily low values, a regime we referred to as geometric exclusion regime (GXR). The extent of the GXR depends on the choice of shape of the front. The $N^{2}$ does vary vertically in the top tens of meters of the ocean, and even though these variations are not likely to be as simple as our exponential decay, constraints may arise, with potential consequences on the probability density functions of Rossby and Richardson numbers.

The combination of DSS and the GXR suggests that we may need to better define the context in which previous studies of pure SI apply. Such studies usually assume that the focus is on the core of the front and that the domains are small enough to relegate lateral variations of the flow to the far field. Current state-of-the-art parameterizations such as that of Bachman et al. (2017) rely on this assumption. However, this reasoning is at odds with DSS in general, and the cases described in sections $5,6 \mathrm{~b}$, and $6 \mathrm{c}$ indicate that this may only be possible in cases that approach the singularity bordering the GXR. Such configurations have been difficult to simulate with our setup. The existence of this singularity may be a hint that such conditions could be hard to find in nature, and at least that this issue requires further investigation.

The idealized nature of this study, and numerical design limitations, highlight multiple avenues of improvement. Perhaps the main limitation of our study lies in its twodimensionality. Recent studies have shown that allowing along-front perturbations drastically modifies the dynamics at play (e.g., Ribstein et al. 2014; Arobone and Sarkar 2015; Natarov and Richards 2015; Skyllingstad et al. 2017), although our main conclusion (i.e., that ISI induces significant PE exchanges) is likely to hold.

A second avenue of improvement would be to better understand the role of the top boundary conditions. In our case, fixing the buoyancy constant on top leads to adiabatic PV fluxes across the surface, which might have influenced the evolution of our instabilities. Some of our experiments featured highly nonlinear growths (not shown) in ways that were hard to predict, and PV fluxes might have been the culprits. However, the question of which set of boundary conditions is the most realistic necessarily depends on the problem (for a discussion on a related problem, see, e.g., Thorpe and Rotunno (1989), Thomas (2005), and Grisouard and Thomas (2016)].

A third avenue of improvement is to better represent the dissipative processes. Our crude representation, based on constant (hyper)viscosity coefficients, has facilitated the observation and interpretations of the experiments, at the expense of accurately representing secondary instabilities (Taylor and Ferrari 2009). Our attempts to detect a quantitative influence of dissipative processes (section 6d) did not yield any robust result but may have been too incomplete to rule them out either. Our main conclusion, which is mainly qualitative, is unlikely to be affected by this omission, since dissipation temporally lags extraction (cf. Fig. 7), but important details about the evolution of the instability might be missing.

And finally, results will be limited as long as no analytical work such as that of Ménesguen et al. (2012) is performed. Such an analysis would allow us to better interpret the results and design the better scalings that are necessary for improved ISI parameterizations. In particular, the framework of Bachman et al. (2017) for pure SI may be amendable to include the impact of LSP and GBP on newly defined operators for momentum and tracer diffusion. While this would represent a significant quantitative expansion of the parameterization, many of the qualitative aspects of pure SI, and in particular the PV flux triggering of SI, would be relatively straightforward to adapt.

Acknowledgments. Fruitful discussions with Riwal Plougonven, Noé Lahaye, Sean Haney, Leif N. Thomas, John R. Taylor, Stephen D. Griffiths, Kelvin J. Richards, Vladimir Zeitlin, Mariona Claret, Pascale Lelong, Scott D. Bachman, and Claire Ménesguen significantly improved this article. Two former undergraduate students, Japinder Nijjer and Morgan B. Fox, helped define the topic of this article in its early stages. Support from the Natural Sciences and Engineering Research Council of Canada (RGPIN-2015-03684) and from the SMAP Canada initiative of the Canadian Space Agency (14SUSWOTTO) is acknowledged. Computations were performed on the GPC supercomputer at the SciNet HPC Consortium. SciNet is funded by the Canada Foundation for Innovation under the auspices of Compute Canada; the Government of Ontario; Ontario Research Fund-Research Excellence; and the University of Toronto (Loken et al. 2010). 


\section{APPENDIX}

\section{Initial Conditions: Intermediate Quantities and Constraints}

Let us first define $\mathrm{Ri}_{0}$, a representative value for $\overline{\mathrm{Ri}}$ with respect to the growth rate of a pure symmetric instability (i.e., its minimum value). This value will be met at the surface of the ocean, where $\overline{\mathrm{Ri}}$ is minimum (because of the simple nature of our front, there is only one such location; see Fig. 1). The expressions for $N^{2}, M^{2}$, and $\overline{\mathrm{Ri}}$ at $z=0$, following the notations of section 2 and introducing $\xi=x / \chi$, are therefore

$$
\begin{aligned}
& \left.N^{2}\right|_{z=0}=\left.\frac{\partial \bar{b}}{\partial z}\right|_{z=0}=N_{0}^{2}+\frac{B}{\delta} \lambda(\xi), \\
& \left.M^{2}\right|_{z=0}=\left.\frac{\partial \bar{b}}{\partial x}\right|_{z=0}=\frac{B}{\chi} \lambda^{\prime}(\xi), \quad \text { and } \\
& \left.\overline{\operatorname{Ri}}\right|_{z=0}=\left.\frac{f^{2} N^{2}}{M^{4}}\right|_{z=0}=\left(\frac{\chi f N_{0}}{B}\right)^{2} \frac{1+\frac{B}{\delta N_{0}^{2}} \lambda(\xi)}{\left[\lambda^{\prime}(\xi)\right]^{2}} .
\end{aligned}
$$

We do not attempt to locate exactly where $\overline{\mathrm{Ri}}$ is minimum, but instead simply locate where its denominator is maximum [i.e., $\xi_{i}$ such that $\lambda^{\prime \prime}\left(\xi_{i}\right)=0$ ] and use it to define

$$
\mathrm{Ri}_{0}=\left(\frac{\chi f N_{0}}{B a_{1}}\right)^{2}\left(1+\frac{B a_{0}}{\delta N_{0}^{2}}\right),
$$

with $a_{0}=\lambda\left(\xi_{i}\right)$ and $a_{1}=\lambda^{\prime}\left(\xi_{i}\right)$. Although $\mathrm{Ri}_{0}>\min (\overline{\mathrm{Ri}})$, their values are sufficiently close (within a factor of $30 \%$ in our numerical simulations) to treat $\mathrm{Ri}_{0}$ as a good enough value to design numerical simulations. The definition of $\mathrm{Ro}_{0}$ is simpler, based on the expression for $\overline{\mathrm{Ro}}$ at $z=0$ :

$$
\left.\overline{\mathrm{Ro}}\right|_{z=0}=\left.\frac{1}{f} \frac{\partial \bar{v}}{\partial x}\right|_{z=0}=\frac{B \delta}{f^{2} \chi^{2}} \lambda^{\prime \prime}(\xi) .
$$

The location $\xi$ that minimizes $\overline{\mathrm{Ro}}$ and therefore would maximize the growth rate of a pure inertial instability, and which we call $\xi_{o}=x_{o} / \chi$, is the one that satisfies $\lambda^{\prime \prime \prime}\left(\xi_{o}\right)=0$. We introduce $a_{2}=\lambda^{\prime \prime}\left(\xi_{o}\right)<0$, such that

$$
\operatorname{Ro}_{0}=\frac{B \delta a_{2}}{f^{2} \chi^{2}} .
$$

Unlike $\mathrm{Ri}_{0}, \mathrm{Ro}_{0}$ is actually the minimum value of $\overline{\mathrm{Ro}}$ over the field. The nondimensional quantities $\xi_{i}, \xi_{o}, a_{0}, a_{1}$, and $a_{2}$ only depend on the choice of $\lambda$. For example, for the tanh profile used in Fig. $1,\left(\xi_{i}, \xi_{o}\right)=[0,-a \sinh (1 / \sqrt{2})]$ and $\left(a_{0}, a_{1}, a_{2}\right)=\left\{1 / 2,-1 / 2,-\left[\sqrt{2} \cosh ^{3}\left(\xi_{o}\right)\right]^{-1}\right\}$, and for the $1-e^{-|\xi|^{3}}$ profile used in the rest of the article, $\left(\xi_{i}, \xi_{o}\right)=\left[-(2 / 3)^{1 / 3},-(1+\sqrt{7} / 3)^{1 / 3}\right]$ and $\left(a_{0}, a_{1}, a_{2}\right) \approx$ $(0.49,-1.18,-2.06)$.

The design of our numerical experiments requires dimensional values based on prescribed values of $\mathrm{Ri}_{0}$, $\mathrm{Ro}_{0}$, and $\Phi_{0}$. If we set the values for $f$ and $\delta$, as we do in the present article, then we have

$$
\frac{B}{\delta f^{2}}=\frac{\mathrm{Ro}_{0} \Phi_{0}^{2}}{a_{2} \mathrm{Bu}_{0}} \quad \text { and } \quad \frac{\chi}{\delta}=\frac{\Phi_{0}}{\sqrt{\mathrm{Bu}_{0}}}
$$

with

$$
\mathrm{Bu}_{0}=\left(\frac{N_{0} \delta}{f \chi}\right)^{2}=\left(\frac{a_{1}^{2}}{a_{2}^{2}} \mathrm{Ri}_{0} \mathrm{Ro}_{0}-\frac{a_{0}}{a_{2}}\right) \mathrm{Ro}_{0} .
$$

In our experiments, we usually have $\mathrm{Bu}_{0} \ll 1$, although its magnitude never is a defining feature of the dynamics at play. By definition, $\mathrm{Bu}_{0} \geq 0$ and $\mathrm{Ro}_{0}<0$, which highlights a strong constraint on which $\left(\mathrm{Ri}_{0}, \mathrm{Ro}_{0}\right)$ couples are allowed, namely, that they have to satisfy

$$
\mathrm{Ri}_{0} \mathrm{Ro}_{0}<a_{0} a_{2} / a_{1}^{2} .
$$

There is therefore a geometric exclusion regime, which depends on $\lambda$. Its origin can be traced back to Eq. (A1a) and the condition that $N^{2}>0$ everywhere in the domain (i.e., that isopycnals do not cross). To meet this condition is nontrivial when the lateral buoyancy gradients vary in both the horizontal and vertical directions. When the condition above is marginally violated $\left(\mathrm{Ri}_{0} \mathrm{Ro}_{0}=a_{0} a_{2} / a_{1}^{2}\right)$, the front has to be infinitely wide to accommodate both a strong lateral density gradient (small $\mathrm{Ri}_{0}$ ) and a weak lateral velocity gradient (small $\left.\mathrm{Ro}_{0}\right)$.

From a practical point of view, we want $a_{0} a_{2} / a_{1}^{2}$ to be as close to zero as possible to minimize the size of the exclusion regime, which partially dictates the choice for $\lambda$. For example, the tanh profile used in Fig. 1 and $\mathrm{e}^{-x^{3}}$ used in the rest of the article are characterized by $a_{0} a_{2} / a_{1} \approx-0.77$ and -0.72 , respectively (recall that $\mathrm{Ro}_{0}<0$ ).

Finally, note that the geometric exclusion regime is a direct consequence of the structure of the front. Indeed, if the frontal part in $\bar{b}$ [Eq. (11)] does not depend on $z$, then $N^{2}=N_{0}^{2}$ everywhere; the parameter $a_{0}$ does not appear in Eqs. (A1c) and (A2), which propagates all the way to Eq. (A7), and the latter is automatically satisfied. On the other hand, if $\chi \rightarrow \infty, \overline{\mathrm{Ro}}, \overline{\mathrm{Bu}} \equiv 0$, and there is no singularity.

\section{REFERENCES}

Arobone, E., and S. Sarkar, 2015: Effects of three-dimensionality on instability and turbulence in a frontal zone. J. Fluid Mech., 784, 252-273, https://doi.org/10.1017/jfm.2015.564. 
Bachman, S. D., and J. R. Taylor, 2014: Modelling of partiallyresolved oceanic symmetric instability. Ocean Modell., 82, 15-27, https://doi.org/10.1016/j.ocemod.2014.07.006.

— B. Fox-Kemper, J. R. Taylor, and L. N. Thomas, 2017: Parameterization of frontal symmetric instabilities. I: Theory for resolved fronts. Ocean Modell., 109, 72-95, https://doi.org/ 10.1016/j.ocemod.2016.12.003.

Boccaletti, G., R. Ferrari, and B. Fox-Kemper, 2007: Mixed layer instabilities and restratification. J. Phys. Oceanogr., 37, 22282250, https://doi.org/10.1175/JPO3101.1.

Callies, J., R. Ferrari, J. M. Klymak, and J. Gula, 2015: Seasonality in submesoscale turbulence. Nat. Commun., 6, 6862, https:// doi.org/10.1038/ncomms 7862 .

Capet, X., J. C. McWilliams, M. J. Molemaker, and F. Shchepetkin, 2008: Mesoscale to submesoscale transition in the California Current system. Part III: Energy balance and flux. J. Phys. Oceanogr., 38, 2256-2269, https://doi.org/10.1175/ 2008JPO3810.1.

Colin de Verdière, A., 2012: The stability of short symmetric internal waves on sloping fronts: Beyond the traditional approximation. J. Phys. Oceanogr., 42, 459-475, https://doi.org/ 10.1175/JPO-D-11-067.1.

_- and R. Schopp, 1994: Flows in a rotating spherical shell: The equatorial case. J. Fluid Mech., 276, 233-260, https://doi.org/ 10.1017/S0022112094002545.

Cushman-Roisin, B., and J.-M. Beckers, 2011: Introduction to Geophysical Dynamics. 2nd ed. Academic Press, 875 pp.

D'Asaro, E., C. Lee, L. Rainville, R. Harcourt, and L. Thomas, 2011: Enhanced turbulence and energy dissipation at ocean fronts. Science, 332, 318-322, https://doi.org/10.1126/ science. 1201515.

D'Orgeville, M., and B. L. Hua, 2005: Equatorial inertialparametric instability of zonally symmetric oscillating shear flows. J. Fluid Mech., 531, 261-291, https://doi.org/10.1017/ S0022112005004015.

Dunkerton, T. J., 1981: On the inertial stability of the equatorial middle atmosphere. J. Atmos. Sci., 38, 2354-2364, https://doi.org/ 10.1175/1520-0469(1981)038<2354:OTISFT $>2.0 . C O ; 2$.

Ferrari, R., and C. Wunsch, 2009: Ocean circulation kinetic energy: Reservoirs, sources, and sinks. Annu. Rev. Fluid Mech., 41, 253-282, https://doi.org/10.1146/annurev.fluid.40.111406. 102139.

Griffiths, S. D., 2003a: Nonlinear vertical scale selection in equatorial inertial instability. J. Atmos. Sci, 60, 977-990, https://doi.org/ 10.1175/1520-0469(2003)060<0977:NVSSIE $>2.0 . C O ; 2$.

- 2003b: The nonlinear evolution of zonally symmetric equatorial inertial instability. J. Fluid Mech., 474, 245-273, https:// doi.org/10.1017/S0022112002002586.

_ 2008: Weakly diffusive vertical scale selection for the inertial instability of an arbitrary shear flow. J. Fluid Mech., 594, 265268.

— 2017: The limiting form of symmetric instability in geophysical flows. Proc. 19th EGU General Assembly, Vienna, Austria, European Geophysical Union, EGU2017-16558, http:// meetingorganizer.copernicus.org/EGU2017/EGU2017-16558.pdf.

Grisouard, N., and L. N. Thomas, 2016: Energy exchanges between density fronts and near-inertial waves reflecting off the ocean surface. J. Phys. Oceanogr., 46, 501-516, https://doi.org/ 10.1175/JPO-D-15-0072.1.

, M. B. Fox, and J. Nijjer, 2016: Radiation of internal waves by symmetrically unstable fronts. Eighth Int. Symp. on Stratified Flows, San Diego, CA, University of California, San Diego, 1-8, http://escholarship.org/uc/item/2b59h10g.
Haine, T. W. N., and J. Marshall, 1998: Gravitational, symmetric, and baroclinic instability of the ocean mixed layer. J. Phys. Oceanogr., 28, 634-658, https://doi.org/10.1175/1520-0485(1998)028<0634: GSABIO $>2.0 . \mathrm{CO} ; 2$.

Holmes, R. M., L. N. Thomas, L. Thompson, and D. Darr, 2014: Potential vorticity dynamics of tropical instability vortices. J. Phys. Oceanogr., 44, 995-1011, https://doi.org/10.1175/ JPO-D-13-0157.1.

Hoskins, B. J., 1975: The geostrophic momentum approximation and the semi-geostrophic equations. J. Atmos. Sci., 32, 233-242, https:// doi.org/10.1175/1520-0469(1975)032<0233:TGMAAT>2.0.CO;2.

Kloosterziel, R. C., and G. F. Carnevale, 2008: Vertical scale selection in inertial instability. J. Fluid Mech., 594, 249-269, https://doi.org/10.1017/S0022112007009007.

Ledwell, J. R., A. J. Watson, and C. S. Law, 1993: Evidence for slow mixing across the pycnocline from an open-ocean tracer-release experiment. Nature, 364, 701-703, https://doi.org/10.1038/364701a0.

,$- \ldots$, and -1998 : Mixing of a tracer in the pycnocline. J. Geophys. Res., 103, 21 499-21 529, https://doi.org/10.1029/ 98JC01738.

Lelong, M.-P., and T. J. Dunkerton, 1998: Inertia-gravity wave breaking in three dimensions. Part II: Convectively unstable waves. J. Atmos. Sci., 55, 2489-2501, https://doi.org/10.1175/ 1520-0469(1998)055<2489:IGWBIT>2.0.CO;2.

Loken, C., and Coauthors, 2010: SciNet: Lessons learned from building a power-efficient top-20 system and data centre. J. Phys. Conf. Ser., 256, 012026, https://doi.org/10.1088/ 1742-6596/256/1/012026.

McWilliams, J. C., 2016: Submesoscale currents in the ocean. Proc. Roy. Soc. London, 472A, 20160117, https://doi.org/10.1098/ rspa.2016.0117.

Ménesguen, C., J. C. McWilliams, and M. J. Molemaker, 2012: Ageostrophic instability in a rotating stratified interior jet. J. Fluid Mech., 711, 599-619, https://doi.org/10.1017/jfm.2012.412.

Molemaker, M. J., J. C. McWilliams, and X. Capet, 2010: Balanced and unbalanced routes to dissipation in an equilibrated Eady flow. J. Fluid Mech., 654, 35-63, https://doi.org/10.1017/ S0022112009993272.

Natarov, A., and K. J. Richards, 2015: Persistent presence of small vertical scale velocity features during three-dimensional equilibration of equatorial inertial instability. Phys. Fluids, 27, 084109, https://doi.org/10.1063/1.4928319.

Plougonven, R., and V. Zeitlin, 2009: Nonlinear development of inertial instability in a barotropic shear. Phys. Fluids, 21, 106601, https://doi.org/10.1063/1.3242283.

Ribstein, B., R. Plougonven, and V. Zeitlin, 2014: Inertial versus baroclinic instability of the Bickley jet in continuously stratified rotating fluid. J. Fluid Mech., 743, 1-31, https://doi.org/ 10.1017/jfm.2014.26.

Skyllingstad, E. D., J. Duncombe, and R. M. Samelson, 2017: Baroclinic frontal instabilities and turbulent mixing in the surface boundary layer. Part II: Forced simulations. J. Phys. Oceanogr., 47, 2429-2454, https://doi.org/10.1175/JPO-D-16-0179.1.

Stone, P. H., 1966: On non-geostrophic baroclinic stability. J. Atmos. Sci., 23, 390-400, https://doi.org/10.1175/1520-0469(1966)023<0390: ONGBS $>2.0 . \mathrm{CO} ; 2$.

Taylor, J. R., and R. Ferrari, 2009: On the equilibration of a symmetrically unstable front via a secondary shear instability. J. Fluid Mech., 622, 103-113, https://doi.org/10.1017/ S0022112008005272.

Thomas, L. N., 2005: Destruction of potential vorticity by winds. J. Phys. Oceanogr., 35, 2457-2466, https://doi.org/10.1175/ JPO2830.1. 
- J. R. Taylor, R. Ferrari, and T. M. Joyce, 2013: Symmetric instability in the Gulf Stream. Deep-Sea Res. II, 91, 96-110, https://doi.org/10.1016/j.dsr2.2013.02.025.

Thorpe, A. S., and R. Rotunno, 1989: Nonlinear aspects of symmetric instability. J. Atmos. Sci., 46, 1285-1299, https://doi.org/ 10.1175/1520-0469(1989)046<1285:NAOSI>2.0.CO;2.

Vallis, G. K., 2006: Atmospheric and Oceanic Fluid Dynamics. Cambridge University Press, 745 pp.

Wang, P., J. C. McWilliams, and C. Ménesguen, 2014: Ageostrophic instability in rotating, stratified interior vertical shear flows. J. Fluid Mech., 755, 397-428, https://doi.org/10.1017/jfm.2014.426.

Whitt, D. B., and L. N. Thomas, 2013: Near-inertial waves in strongly baroclinic currents. J. Phys. Oceanogr., 43, 706-725, https://doi.org/10.1175/JPO-D-12-0132.1.

- and —_, 2015: Resonant generation and energetics of wind-forced near-inertial motions in a geostrophic flow.
J. Phys. Oceanogr., 45, 181-208, https://doi.org/10.1175/ JPO-D-14-0168.1.

Winters, K. B., and J. J. Riley, 1992: Instability of internal waves near a critical level. Dyn. Atmos. Oceans, 16, 249-278, https:// doi.org/10.1016/0377-0265(92)90009-I.

—, and E. A. D'Asaro, 1994: Three-dimensional wave instability near a critical level. J. Fluid Mech., 272, 255-284, https://doi.org/ 10.1017/S0022112094004465.

__ and A. de la Fuente, 2012: Modelling rotating stratified flows at laboratory-scale using spectrally-based DNS. Ocean Modell., 49-50, 47-59, https://doi.org/10.1016/ j.ocemod.2012.04.001.

- J. A. MacKinnon, and B. Mills, 2004: A spectral model for process studies of rotating, density-stratified flows. J. Atmos. Oceanic Technol., 21, 69-94, https://doi.org/ 10.1175/1520-0426(2004)021<0069:ASMFPS>2.0.CO;2. 